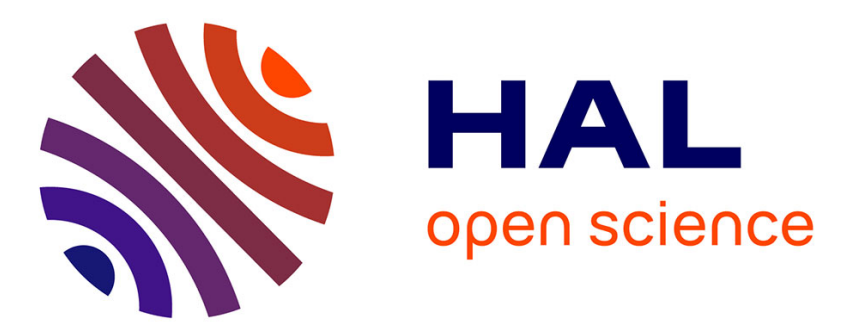

\title{
Sensitivity analysis of non-local damage in soft biological tissues
}

\author{
Di Zuo, Stéphane Avril, Chunjiang Ran, Haitian Yang, S. Jamaleddin \\ Mousavi, Klaus Hackl, Yiqian He
}

\section{- To cite this version:}

Di Zuo, Stéphane Avril, Chunjiang Ran, Haitian Yang, S. Jamaleddin Mousavi, et al.. Sensitivity analysis of non-local damage in soft biological tissues. International Journal for Numerical Methods in Biomedical Engineering, inPress, 10.1002/cnm.3427 . hal-03139932

\section{HAL Id: hal-03139932 \\ https://hal.science/hal-03139932}

Submitted on 12 Feb 2021

HAL is a multi-disciplinary open access archive for the deposit and dissemination of scientific research documents, whether they are published or not. The documents may come from teaching and research institutions in France or abroad, or from public or private research centers.
L'archive ouverte pluridisciplinaire HAL, est destinée au dépôt et à la diffusion de documents scientifiques de niveau recherche, publiés ou non, émanant des établissements d'enseignement et de recherche français ou étrangers, des laboratoires publics ou privés. 


\title{
Sensitivity analysis of non-local damage in soft biological tissues
}

\author{
Di Zuo ${ }^{\mathrm{a}}$, Stéphane Avril ${ }^{\mathrm{b}}$, Chunjiang Ran ${ }^{\mathrm{a}}$, Haitian Yang ${ }^{\mathrm{a}}$, S. Jamaleddin Mousavi ${ }^{\mathrm{b}}$, Klaus \\ Hackl ${ }^{\mathrm{c}}$, Yiqian $\mathrm{He}^{\mathrm{a}, \mathrm{d}, *}$ \\ ${ }^{a}$ State Key Lab of Structural Analysis for Industrial Equipment, Department of Engineering Mechanics, \\ Dalian University of Technology, Dalian 116024, P.R. China \\ ${ }^{b}$ Mines Saint-Étienne, University Lyon, INSERM, U1059 Sainbiose, Centre CIS, Saint-Étienne F-42023, \\ France \\ ${ }^{c}$ Institute of Mechanics of Materials, Ruhr-Universität Bochum, Bochum D-44801, Germany \\ ${ }^{d}$ Key Laboratory of Biorheological and Technology of Ministry of Education, Chongqing University, \\ Chongqing 400030, P. R. China
}

\begin{abstract}
Computational modeling can provide insight into understanding the damage mechanisms of soft biological tissues. Our gradient-enhanced damage model presented in a previous publication has shown advantages in considering the internal length scales and in satisfying mesh independence for simulating damage, growth and remodeling processes. Performing sensitivity analyses for this model is an essential step towards applications in which it is necessary to handle the uncertainty caused by patient-specific data. In this paper, a numerical analysis approach is developed by integrating two existing methods, i.e. the gradient-enhanced damage model and the surrogate model-based probability analysis, to address this need. To increase the computational efficiency of the Monte Carlo method in uncertainty propagation for the nonlinear hyperelastic damage analysis, the surrogate model based on Legendre polynomial series is employed to replace the direct FEM solutions, and the sparse grid collocation method (SGCM) is adopted for setting the collocation points to further reduce the computational cost in training the surrogate model. The effectiveness of the proposed approach is illustrated by two numerical examples, including an application to the clinical problem of dilatation of the artery.
\end{abstract}

Keywords: Nonlocal damage, Uncertainty analysis, Soft tissues, Surrogate model, Computational efficiency

\section{Introduction}

Understanding the damage mechanism of soft biological tissues is critical to the characterization of tissue injuries [1, 2, 3, 4]. For instance, in the treatment of cardiovascular 4 diseases, the focus is often on the potential risk of tissue rupture, which is mainly caused by 5 soft tissue damage, such as atherosclerotic plaques or aneurysms [5]. The damage analyses

*Corresponding author:heyiqian@dlut.edu.cn 
of the tendons, which are important load-bearing structures and are frequently injured in both sports and work, are also important in understanding the underlying mechanisms and are helpful for treatment [6].

The damage modeling of soft biological tissues and its numerical computation has been a topic of intense research. Holzapfel et al. [7], Gasser [8] and Li [9] comprehensively reviewed the computational models for the damage of soft biological tissues. The developed damage models can be divided into three approaches [9]: (1) models based on continuum damage mechanics, (2) models based on the theory of pseudo-elasticity, and (3) the softening hyperelasticity approach. Among the continuum damage models, a gradient-enhanced non-local damage model was proposed by Dimitrijevic and Hackl [10, 11, and a nonlocal continuum healing model that combines the gradient-enhanced damage model and a temporally homogenized growth and remodeling model was originally presented in our previous works [12, 13]. This model has the following advantages: (1) good mesh independence is achieved in the simulation of damage evolution with growth and remodeling; and (2) the nonlocal damage process is realized by introducing the gradient-enhanced variable, thus allowing the effect of internal length scales of soft tissues to be considered.

Uncertainty widely exists due to the variability in human tissues, such as the patientdependent structural uncertainty and parametric uncertainty [14]. Parameter sensitivity analysis involving uncertainty is an essential step for developing and applying damage models to soft biological tissues. For instance, parameter sensitivity is the key information for medical device approval [15]. Some probabilistic method-based models have been presented to analyze the uncertainty of biomechanical problems. For instance, Laz and Browne [16] presented an overview of probability analysis in structural reliability, kinematics, joint mechanics, musculoskeletal modeling, and patient-specific representations. Regarding the uncertain damage analysis of soft tissues, Rodríguez et al. [17 presented a model taking into account a stochastically-distributed waviness and a subsequent rupture of the individual fibers as the main driving force for damage evolution, and Schmidt et al. [18] proposed a statistical approach to describe the evolution of microscopic damage in soft collagenous tissues. More recently, Balzani et al. [19] introduced a computational method for the assessment of rupture probabilities in soft collagenous tissues based on the numerical minimization and maximization of the probability of failure $(\mathrm{PoF})$, which arise from random input quantities. Although the above models have been proposed to deal with the uncertainty analysis in biomechanics, there is still no work on the analysis of the uncertainty in the nonlocal damage model for soft biological tissues.

The large computational cost is a main challenge in uncertainty analysis when a probabilistic method is used [19, 20]. Although the Monte Carlo method is the most straightforward way to conduct probability analysis [21, deterministic problems must usually be solved many times to guarantee the accuracy of the Monte Carlo method. Especially for the damage analysis for soft biological tissues, the computation of one deterministic problem usually requires considerable computational cost due to the solutions of nonlinear problems. Therefore, it is desirable to reduce the computational cost of uncertain damage analysis for soft biological tissues.

The surrogate model is an effective approach to reduce the computational cost of uncer- 
tainty analysis 22. The key idea of the surrogate model is to use an approximate model with rather a simple form to replace the original model by fitting the relationship between the input and output variables [23]. An overview of surrogate models can be found in the literatures provided by Díaz-Manríquez et al. [24], Razavi et al. [25], Asher et al. [26], Zhang [27] or Raul et al. [28]. The widely-used surrogate models include the following: polynomial approximation models [29], the radial basis function [30, Kriging models [31], etc. In this paper, the Legendre polynomial series surrogate model proposed by Wang et al. [32] is employed in the damage analysis of soft tissues due to its excellent error properties in the approximation of a globally smooth function [33].

To investigate the sensitivity of the uncertain material properties for the damage analysis of soft tissues, a numerical analysis approach is developed by integrating the gradientenhanced damage model [10, 11] and the Legendre polynomial series surrogate model [32] introduced above. The gradient-enhanced damage model is adopted to describe the behavior of the stress-softening of soft tissues and avoid mesh-dependence. The Legendre polynomial is used to replace the finite element method (FEM) solutions of deterministic problems, and the sparse grid collocation method (SGCM) is employed because of its advantage of using fewer collocation points when training surrogate models.

The remainder of this paper is organized as follows: Section 2 introduces the materials and methods, including the basic kinematics in Section 2.1, the gradient-enhanced damaged model in Section 2.2. the total potential energy and variational form in Section 2.3, the constitutive model in Section 2.4, the surrogate model in Section 2.5 and probability analysis in Section 2.6. Section 3 shows two numerical examples. Finally, discussions and conclusions are given in Section 4 .

\section{Methods and materials}

\subsection{Basic kinematics}

Let $\boldsymbol{x}=\boldsymbol{\varphi}(\boldsymbol{X}, t)$ describe the motion of a body from the initial reference configuration $\boldsymbol{X} \in \kappa(0)$ to its current configuration $\boldsymbol{x} \in \kappa(t)$. The deformation gradient $\boldsymbol{F}$ and the Jacobian $J$ that maps the referential volume $\mathrm{d} V$ onto the current volume $\mathrm{d} v$ are defined as

$$
\begin{aligned}
& \boldsymbol{F}=\nabla_{\boldsymbol{X}} \boldsymbol{\varphi}, \\
& J=\frac{\mathrm{d} v}{\mathrm{~d} V}=\operatorname{det}(\boldsymbol{F}) .
\end{aligned}
$$

\subsection{Gradient-enhanced damage model}

The local strain energy function $\Psi^{\text {loc }}$ per unit of reference volume at each time is written as

$$
\Psi^{\mathrm{loc}}=f(d) \Psi_{0},
$$


where $\Psi_{0}$ is the original (undamaged) strain energy density and $f(d)$ represents a function of the damage variable $d$ that measures the degree of material stiffness loss. In Eq. (3), $f(d)=1$ means there is no damage and the total damage for $f(d) \rightarrow 0$. It is at least twice differentiable and satisfies the following conditions:

$$
f(d) \quad: \quad \Re^{+} \rightarrow(0,1]\left\{f(0)=1, \lim _{d \rightarrow \infty}^{f}(d)=0\right\} .
$$

Following the approaches of Dimitrijevic and Hackl [10, 11], a gradient-enhanced nonlocal free energy term is added to the strain energy given in Eq. (3)

$$
\Psi=f(d) \Psi_{0}+\frac{c_{\mathrm{d}}}{2}\left\|\nabla_{\boldsymbol{X}} \phi\right\|^{2}+\frac{\beta_{\mathrm{d}}}{2}\left[\phi-\gamma_{\mathrm{d}} d\right]^{2} .
$$

In Eq. (5), $c_{\mathrm{d}}$ represents the gradient parameter that defines the degree of gradient regularization and the internal length scales. Three other variables are introduced:

- the field variable $\phi$, which transfers the values of the damage parameter across the element boundaries to make it non-local by nature;

- the energy-related penalty parameter $\beta_{\mathrm{d}}$, which approximately forces the local damage field and the nonlocal field to coincide; and

- parameter $\gamma_{d}$, which is set as a switch between the local and enhanced models. When $\gamma_{d}=0$ and $c_{d}=0$, it leads to a local model, while setting $\gamma_{d}=1$ and $c_{d} \neq 0$ leads to the enhanced non-local model. A typical value of this parameter is set to 1 following Dimitrijevic and Hackl [10, 11] and Waffenschmidt et al. 34.

\subsection{Total potential energy and variational form}

The general total potential energy for nonlocal damage model is

$$
\Pi=\int_{\Omega} \Psi \mathrm{d} V-\int_{\Omega} \overline{\boldsymbol{B}} \cdot \boldsymbol{\varphi} \mathrm{d} V-\int_{\partial \Omega} \overline{\boldsymbol{T}} \cdot \boldsymbol{\varphi} \mathrm{d} V,
$$

where $\overline{\boldsymbol{B}}$ is the body force vector per unit of reference volume of $\Omega$ and $\overline{\boldsymbol{T}}$ is the traction on the boundary $\partial \Omega$.

Minimization of the potential energy with respect to the primal variables $\varphi$ and $\phi$ results in a coupled non-linear system of equations, which may be written as

$$
\begin{aligned}
& \int_{\Omega} \boldsymbol{P}: \nabla_{\boldsymbol{X}} \delta \boldsymbol{\varphi} \mathrm{d} V-\int_{\Omega} \overline{\boldsymbol{B}} \cdot \delta \boldsymbol{\varphi} \mathrm{d} V-\int_{\partial \Omega} \overline{\boldsymbol{T}} \cdot \delta \boldsymbol{\varphi} \mathrm{d} V=0, \\
& \int_{\Omega} \boldsymbol{Y}: \nabla_{\boldsymbol{X}} \delta \phi \mathrm{d} V-\int_{\Omega} Y \delta \phi \mathrm{d} V=0,
\end{aligned}
$$

where $\boldsymbol{P}$ is the first Piola-Kirchhoff stress, $\boldsymbol{Y}$ is vectorial damage quantity related to flux terms and $Y$ is the scalar damage quantity associated to source terms. They are defined as

$$
\boldsymbol{P}=\partial_{\boldsymbol{F}} \Psi, \quad \boldsymbol{Y}=\partial_{\nabla_{\boldsymbol{X}} \phi} \Psi, \quad Y=-\partial_{\phi} \Psi .
$$



105 by

$$
\begin{array}{ll}
\boldsymbol{\sigma}=\boldsymbol{P} \cdot \operatorname{cof}\left(\boldsymbol{F}^{-1}\right), & \overline{\boldsymbol{b}}=J^{-1} \overline{\boldsymbol{B}}, \\
\boldsymbol{y}=\boldsymbol{Y} \cdot \operatorname{cof}\left(\boldsymbol{F}^{-1}\right), & y=J^{-1} Y,
\end{array}
$$

where $\operatorname{cof}(\boldsymbol{F})=J \boldsymbol{F}^{-\mathrm{T}}$.

Substituting Eqs. (2), (10) and (11) into Eqs. (7) and (8), the variational forms of the spatial description are

$$
\begin{aligned}
& \int_{\Omega} \boldsymbol{\sigma}: \nabla_{\boldsymbol{x}} \delta \boldsymbol{\varphi} \mathrm{d} v-\int_{\Omega} \overline{\boldsymbol{b}} \cdot \delta \boldsymbol{\varphi} \mathrm{d} v-\int_{\partial \Omega} \overline{\boldsymbol{t}} \cdot \delta \boldsymbol{\varphi} \mathrm{d} v=0, \\
& \int_{\Omega} \boldsymbol{y}: \nabla_{\boldsymbol{x}} \delta \phi \mathrm{d} v-\int_{\Omega} y \delta \phi \mathrm{d} v=0 .
\end{aligned}
$$

\subsection{Constitutive model}

\subsubsection{Hyperelastic part of the free energy}

For the undamaged part $\Psi_{0}$ in Eq. (3), we used the Holzapfel-Gasser-Ogden (HGO) hyperelastic constitutive model [35, 36], which can be written as

$$
\Psi_{0}=\Psi^{\text {iso }}+\Psi^{\text {aniso }},
$$

where the isotropic part $\Psi^{\text {iso }}$ and anisotropic part $\Psi^{\text {aniso }}$ are

$$
\begin{aligned}
& \Psi^{\text {iso }}=\frac{1}{2} \mu_{0} J^{-2 / 3}\left(I_{1}-3\right)+\frac{1}{2} \kappa_{0}(J-1)^{2}, \\
& \Psi^{\text {aniso }}=\frac{k_{1}}{2 k_{2}} \sum_{i=4,6}\left[\exp \left(k_{2}<E_{i}>^{2}\right)-1\right],
\end{aligned}
$$

where $\mu_{0}$ and $\kappa_{0}$ are the shear and bulk moduli of the soft isotropic matrix, respectively. $I_{1}=\operatorname{tr}(\boldsymbol{C})$ is the first invariant of $\boldsymbol{C}$, where $\boldsymbol{C}$ is the right Cauchy-Green tensor, which is defined as $\boldsymbol{C}=\boldsymbol{F}^{\mathrm{T}} \boldsymbol{F}$. In Eq. (16), $E_{i}=\varkappa I_{1}+(1-3 \varkappa) I_{4 i}-1$ is a strain-like quantity and the $I_{4 i}=\boldsymbol{C}: \boldsymbol{A}_{i}=\boldsymbol{C}:\left(\boldsymbol{a}_{0 i} \otimes \boldsymbol{a}_{0 i}\right)$ is the fourth invariant (which equals the square of the stretch of collagen fibers), the $\varkappa \in[0,1 / 3]$ is a dispersion parameter and $\boldsymbol{a}_{0 i}=(\cos \theta, \sin \theta, 0)$ is the unit direction vector of the fiber in the reference configuration. $k_{1}$ and $k_{2}$ are material parameters. The term $\left\langle E_{i}>\right.$, where $\left\langle E_{i}\right\rangle=\left(\left|E_{i}\right|+E_{i}\right) / 2$ is the Macaulay bracket, reflects the basic assumption that fibers can only support tension. 


\subsubsection{Damage evolution}

The detailed evolution of the damage variable $d$ can be found in the works of Dimitrijevic and Hackl [10, 11] and Waffenschmidt et al. 34]. In the following, we give a brief description of some basic and essential formulations.

Following standard thermodynamic considerations [10, 11, the damage conjugate driving force $q$ is defined as

$$
q=-\frac{\partial \Psi}{\partial d} .
$$

The damage condition at any time of the loading process is based on an energy release rate threshold condition [37]

$$
\Phi_{\mathrm{d}}=q-r_{1} \leq 0,
$$

where $r_{1}$ represents the threshold value that triggers the evolution of the damage.

The differential equation of the evolution of damage is subjected to the Kuhn-Tucker optimality conditions [10, 11]

$$
\dot{d}=\dot{\kappa} \frac{\partial \Phi_{\mathrm{d}}}{\partial q}, \quad \dot{\kappa} \geq 0, \quad \Phi_{\mathrm{d}} \leq 0, \quad \dot{\kappa} \Phi_{\mathrm{d}}=0,
$$

where $\dot{\kappa}$ is the Lagrange multiplier.

\subsubsection{Verification of the damage model}

An exemplary qualitative simulation is applied here to show how this non-local damage model can capture the stiffness loss of soft tissues when subjected to an overloading beyond physiological loads, and the results are shown in Fig. 1. In this simulation, an exponential damage function $f(d)=e^{-d}$ was adopted and was also used in following simulations. Other damage functions satisfying Eq. (4) could also be used 34. The chosen model was compared with experimental stress-strain responses of Raghavan et al. 38. In these experimental data, thin strips of abdominal aortic aneurysm excised from the anterior surface during surgical repair were tested. Both the elastic and damage phases were well described by the non-local damage model as shown in Fig. 1.

\subsection{Surrogate model}

\subsubsection{Legendre polynomial surrogate model}

In this paper, the Legendre polynomial surrogate model is used to approximate the results of the FEM [32], and the Rodrigues formula of the $N$-order Legendre polynomial $\Phi(\alpha)$ can be written as

$$
\Phi(\alpha)=\frac{1}{2^{N} N !} \frac{d^{N}}{d(\alpha)}\left[\left((\alpha)^{2}-1\right)^{N}\right],
$$

where $\alpha$ is the uncertain variable and $N$ is the order of the polynomial series. 
Based on the polynomial chaos framework [39], the output variable $L(\boldsymbol{x}, \boldsymbol{\alpha})$ can be expressed as

$$
L(\boldsymbol{x}, \boldsymbol{\alpha})=\sum_{\mathbf{i}} w_{\mathbf{i}}(\boldsymbol{x}) \Phi_{\mathbf{i}}(\boldsymbol{\alpha}),
$$

where $\boldsymbol{x}$ is the spatial coordinate, $\boldsymbol{\alpha}$ is the uncertain variables vector, $w_{\mathbf{i}}(\boldsymbol{x})$ is the corresponding expansion coefficient and $\mathbf{i}=\left(i_{1}, i_{2}, \ldots, i_{n}\right)$ stands for the multigauge. Note that $L$ is the output variable, and it can be any variables concerned (stress and damage in this paper). In practice, a maximum order of polynomials $N$ is selected to truncate Eq. (21) up to finite terms, by which Eq. (21) can be concisely rewritten as

$$
L(\boldsymbol{x}, \boldsymbol{\alpha}) \approx L_{N}(\boldsymbol{x}, \boldsymbol{\alpha})=\sum_{\mathbf{i} \leq N} w_{\mathbf{i}}(\boldsymbol{x}) \Phi_{\mathbf{i}}(\boldsymbol{\alpha})
$$

\subsubsection{Sparse grid collocation method ( $S G C M$ )}

The basic idea of a collocation method is using the response values at preselected points in uncertainty space to calculate the expansion coefficients of the surrogate model [32]. In this paper, the SGCM based on the Clenshaw-Curtis point-based collocation method and the Smolyak algorithm is adopted with the advantage that it can minimize the collocation error with the same number of nodes under the maximum norm [32].

For the univariate case, the Clenshaw-Curtis nodes distributed in the interval range $\left[\underline{\alpha_{i}}, \overline{\alpha_{i}}\right]$ are defined as

$$
\beta_{j}^{i}=\left\{\begin{array}{ll}
\alpha_{i}^{c} & \text { if } m_{i}=1 \\
\alpha_{i}^{c}-\cos \frac{\pi(j-1)}{m_{i}-1} \cdot \Delta \alpha_{i} & \text { if } m_{i}>1
\end{array},\right.
$$

where $\alpha_{i}^{c}=\left(\bar{\alpha}_{i}+\underline{\alpha}_{i}\right) / 2, \Delta \alpha_{i}=\left(\bar{\alpha}_{i}-\underline{\alpha}_{i}\right) / 2$, in which $\bar{\alpha}$ and $\underline{\alpha}$ represents the upper bound and lower bound of the uncertain interval, respectively, $m_{i}$ is the predefined number of collocation points.

For the multivariate case, the $n$ input parameters are assumed to be independent with each other for simplification. The general approach is to extend the collocation points defined in one dimension in Eq. (23) to the entire interval uncertain space using a tensor product. For each dimension, a nodal set $\Theta_{i}^{m_{i}}=\left\{\beta_{1}^{i}, \beta_{2}^{i}, \ldots, \beta_{m_{i}}^{i}\right\}$ is selected. On this basis, the entire nodal set named the full tensor product grids can be denoted as

$$
\boldsymbol{\Theta}=\boldsymbol{\Theta}_{1}^{m_{1}} \times \Theta_{2}^{m_{2}} \times \ldots \times \Theta_{n}^{m_{n}},
$$

where the total number of collocation points $M$ is

$$
M=\prod_{i=1}^{n} m_{i}=m_{1} \times m_{2} \times \ldots \times m_{i} .
$$

To reduce the huge computational cost for high-dimensional problems, Smolyak-type sparse grids [40] are introduced, which are still based on the tensor product construction, 
but only a small subset of the full tensor grids Eq. (24) are selected. By using the Smolyak algorithm, the total nodal set of collocation points in Eq. (24) can be reconstructed in the following form:

$$
\boldsymbol{\Theta}=\bigcup_{l-n+1 \leq \mathbf{i} \mid \leq l}\left(\boldsymbol{\Theta}_{1}^{i_{1}} \times \boldsymbol{\Theta}_{2}^{i_{2}} \times \ldots \times \boldsymbol{\Theta}_{n}^{i_{n}}\right),
$$

where $l \geq n$ is an integer representing the overall level of the construction and $i_{j}$ represents the separate level along the $j$ th direction.

By iterating through all the nodes on full grids, only the ones whose sum order $|\mathbf{i}|=$ $i_{1}+i_{2}+\ldots+i_{n}$ across all dimensions is from $l-n+1$ to $l$ are retained. To define the collocation points, an index $k$ is introduced to denote the level of collocation points in onedimensional uncertainty space. The number $m_{i}^{k}$ and positions $\beta_{j}^{i}$ of the Clenshaw-Curtis nodes in the interval range $\left[\alpha_{i}, \overline{\alpha_{i}}\right]$ are defined as

$$
\begin{aligned}
m_{i}^{k} & = \begin{cases}1 & \text { if } k=1 \\
2^{k-1}+1 & \text { if } k>1\end{cases} \\
\beta_{j}^{i} & =\left\{\begin{array}{ll}
\alpha_{i}^{c} & \text { if } k=1 \\
\alpha_{i}^{c}-\cos \frac{\pi(j-1)}{m_{i}^{k}-1} \cdot \Delta \alpha_{i} \quad j=1,2 \ldots, m_{i}^{k} & \text { if } k>1
\end{array} .\right.
\end{aligned}
$$

After selecting the SGCM nodal set, a set $\boldsymbol{\Theta}=\left\{\boldsymbol{\beta}_{1}, \boldsymbol{\beta}_{2}, \ldots, \boldsymbol{\beta}_{M}\right\}$ is given to specifically denote all the collocation points in the interval uncertain space, and $L\left(\boldsymbol{x}, \boldsymbol{\beta}_{j}\right)$ is the solution of the objective variable in the deterministic problem at the preselected points $\boldsymbol{\beta}_{j}$ that could be obtained by the FEM. Based on the surrogate function in Eq. (22), a group of linear equations with respect to the unknown expansion coefficients $w_{\mathbf{i}}(\boldsymbol{x})$ can be derived as

$$
\left(\begin{array}{cccc}
\Phi_{1}\left(\boldsymbol{\beta}_{1}\right) & \Phi_{2}\left(\boldsymbol{\beta}_{1}\right) & \cdots & \Phi_{C_{n+N}^{n}}\left(\boldsymbol{\beta}_{1}\right) \\
\Phi_{1}\left(\boldsymbol{\beta}_{2}\right) & \Phi_{2}\left(\boldsymbol{\beta}_{2}\right) & \cdots & \Phi_{C_{n+N}^{n}}\left(\boldsymbol{\beta}_{2}\right) \\
\vdots & \vdots & \ddots & \vdots \\
\Phi_{1}\left(\boldsymbol{\beta}_{M}\right) & \Phi_{2}\left(\boldsymbol{\beta}_{M}\right) & \cdots & \Phi_{C_{n+N}^{n}}\left(\boldsymbol{\beta}_{M}\right)
\end{array}\right)\left(\begin{array}{c}
w_{1}(\boldsymbol{x}) \\
w_{2}(\boldsymbol{x}) \\
\vdots \\
w_{C_{n+N}^{n}}(\boldsymbol{x})
\end{array}\right)=\left(\begin{array}{c}
L\left(\boldsymbol{x}, \beta_{1}\right) \\
L\left(\boldsymbol{x}, \beta_{2}\right) \\
\vdots \\
L\left(\boldsymbol{x}, \beta_{M}\right)
\end{array}\right) .
$$

Once the polynomial bases $\Phi_{\mathbf{i}}$ and the SGCM nodal set $\boldsymbol{\Theta}$ are given, the expansion coefficients $w_{\mathbf{i}}(\boldsymbol{x})$ in Eq. (22) can be solved by Eq. (28). Note that the number of collocation points is required not to be smaller than the number of polynomial expansion terms, such as $M \geq C_{n+N}^{n}$, to prevent the problem from being undetermined.

\subsection{Probability analysis}

The prediction of the rupture of soft tissues with uncertain parameters can be captured by the rupture probability, and $\mathrm{PoF}$ is defined as the probability that a mechanical rupture criterion holds. Such a criterion can be formulated as the inequality $f>f^{\max }$, where $f$ represents a mechanical quantity of interest, for example, the stress, strain, or damage, which is considered relevant for initializing a rupture [19]. 
Let $\boldsymbol{R}$ be a set of input parameters and $z(\boldsymbol{R})$ is the mechanical quantity of interest. A suitable failure criterion can be defined as

$$
z(\boldsymbol{R}) \geq z^{\max },
$$

where $z^{\max }$ is a maximally admissible value. Generally, the PoF can be defined as

$$
\mathrm{PoF}:=\mathbb{P}\left[z(\boldsymbol{R}) \geq z^{\max }\right]
$$

where $\mathbb{P}(\cdot)$ is the probability of $(\cdot)$.

In this paper, the beta distribution is used for uncertain parameters to avoid some possible unreasonable values of the distribution, that could be produced in a Gaussian distribution [18]. The probability density function (PDF) of the beta distribution is defined as [41]:

$$
\operatorname{PDF}(\alpha ; a, b)=\frac{1}{B(a, b)} \alpha^{a-1}(1-\alpha)^{b-1},
$$

where $\alpha$ is the uncertain parameter, $a$ and $b$ denote two shape parameters and $B(\cdot)$ is the beta function. Note that some alternative distributions, e.g. the lognormal distribution [42], could also be used for uncertain parameters.

In this paper, the damage function $f(d)$ is used to describe the risk of the rupture, and a lower value of $f(d)$ means a higher risk of rupture. Here, the PoF is redefined as

$$
\mathrm{PoF}:=\mathbb{P}\left[f(d) \leq f(d)^{\max }\right] .
$$

The PoF can be calculated as the integral of the PDF of the damage function $f(d)$ from $f(d)=0$ up to $f(d)=f(d)^{\max }$ as

$$
\mathrm{PoF}:=\mathbb{P}\left[f(d) \leq f(d)^{\max }\right]=\int_{0}^{f(d)^{\max }} \operatorname{PDF}(f(d)) \mathrm{d} f(d) .
$$

An example is shown for the computation of the PoF in Fig. 2. The damage function is assumed to obey a beta distribution as $f(d) \sim B(4,4)$ within the interval $(0,1]$ and the maximum damage is set to $f(d)^{\max }=0.5$. With these assumptions, the value of the PoF can be calculated as 0.5 according Eq. (33), which is equaling to the area of gray part in Fig. 2.

\section{Numerical examples}

The gradient-enhanced damage model is implemented within the commercial finite element software Abaqus/Standard by means of a user subroutine UEL and the simulation of the probability analyses based on the surrogate model are carried out by Matlab R2018a. Both computations are conducted on a PC with an Intel Xeon E5-2650 CPU @ $2.40 \mathrm{GHz}$ and 32 GB of RAM. 
The first example is used to illustrate the nonlocal damage model and the advantages of the surrogate model with the SGCM through an open-hole plate case. The second example is a radial dilatation of an idealized artery, which is used to treat atherosclerotic plaque. Note that only homogeneous uncertain material parameters without spatial correlation are assumed for simplification, and plane strain elements (Bilinear 8 node element CPE8 in Abaqus) are used in all simulations.

\subsection{Open-hole plate}

The first numerical example is an open-hole plate under displacement loading. The geometry and the FEM model are shown in Fig. 3. Due to the symmetry, only one-fourth of the plate is analyzed. In this example, only the isotropic contribution of the soft tissues is considered. The result of the deterministic analysis are shown in Section 3.1.1, the verification of the surrogate model is shown in Section 3.1 .2 and the results of the probability analysis are shown in Section 3.1.3.

\subsubsection{Deterministic analysis}

In this part, a series tests on 286 elements are performed, which the mesh-dependence had been already examined in our previous works [12, 13], to investigate the influence of the shear modulus and the internal length scales on the damage analysis in deterministic problem. The geometry, hyperelastic and damage parameters are reported in Table 1, in which the bulk modulus $k_{0}$ is set to 100 times of the shear modulus to make the material nearly incompressible.

The contours of the damage function $f(d)$ for different shear modulus are shown in Fig. 4 , while the internal length scales is $c_{\mathrm{d}}=1.0 \mathrm{MPa}^{-1} \cdot \mathrm{mm}^{2}$. It can be obviously seen that a larger value of the shear modulus resulting a larger damage, and the same results can be also observed in Fig. 5. The value of the parameter $c_{d}$ represents the degree of regularization and it depends on the microstructure of the tissues. The influence of the internal length scales $c_{d}$ is investigated by three different values and the results are shown in Figs. 6 and 7 , while the shear modulus is a constant that $\mu_{e}=0.1 \mathrm{MPa}$. As shown in Figs. 6 and 7 , the levels of damage are strongly depending on the value of $c_{d}$. It can be explained by the width of the activated zone [10, 11, 34]. With a smaller $c_{d}$, a smaller region is influenced by the damage variable, resulting in more concentrated distribution of the damage.

\subsubsection{Verification of the surrogate model}

In the training of the surrogate model, the 5-order Legendre polynomial series and the SGCM with $k=4$ are used. The comparison of the values of $f(d)$ and $\sigma_{x}$ computed by the surrogate model and the FEM at six feature nodes (the locations are shown in Fig. 3(b) for Case 1 when the stretch is $\lambda_{x}=0.1$ are listed in Table 3. A good agreement can be seen in Table 3 such that the surrogate model with the SGCM can approximate the results of the FEM with the maximum difference being less than $1 \%$. The good performance of the surrogate model with the SGCM is addressed again by comparing the results of the surrogate model with the SGCM and FEM at Node 1 when $\lambda_{x}=0.1$ for Case 2 and Case 3 , and the results are shown in Fig. 8 . 


\subsubsection{Probability analysis}

In this example, shear modulus $\mu_{\mathrm{e}}$ and internal length scales $c_{\mathrm{d}}$ are assumed to be uncertain parameters that obey the beta distributions in the given intervals $\left(\mu_{\mathrm{e}} \in[0.07,0.13]\right.$ $\mathrm{MPa}$ and $\left.c_{\mathrm{d}} \in[0.5,1.5] \mathrm{MPa}^{-1} \cdot \mathrm{mm}^{2}\right)$, and seven cases for different levels of uncertainty for $\mu_{\mathrm{e}}$ and $c_{\mathrm{d}}$ listed in Table 2 are analyzed. The bulk modulus $k_{0}$ is set to 100 times of the mean value of the shear modulus to make the material nearly incompressible. Apart from $\mu_{e}, c_{d}$ and $k_{0}$, other geometric and material parameters are shown in Table 1 .

First, we investigate the computational cost for using the Monte Carlo method based on the direct FEM and the Monte Carlo method based on the surrogate model. When employing the Monte Carlo method, $10^{5}$ samples are computed to ensure the accuracy of the probability analysis, as suggested by Wu et al. [43. The comparison of the CPU times is shown in Table 4. Note that the computational time for the Monte Carlo method with the direct FEM is estimated by multiplying the time of one FEM by $10^{5}$. A significant reduction of the computational cost can be observed in Table 4 such that the computing time of the Monte Carlo method with the surrogate model is about $0.06 \%$ of that using the Monte Carlo method with the direct FEM but the relative error is less than $1 \%$.

Second, the individual and combined impacts of the uncertain parameters on the evolution of the damage are investigated through three different cases and the PDFs of the damage function $f(d)$ at Node 3 (the location is shown in Fig. 3(b) are shown in Fig. 9(a), Comparing Case 1 and 2, the mean value $\hat{\mu}$ of the damage function $f(d)$ is close but the standard deviation $\hat{\sigma}$ for Case 1 is larger than that for Case 2, which means that the shear modulus $\mu_{\mathrm{e}}$ has a relatively larger influence on $f(d)$ than the internal length scales $c_{\mathrm{d}}$ in this example. For Case 3, a larger standard deviation $\hat{\sigma}=0.087$ can be found as expected and there is little difference in the mean value $\hat{\mu}$ between Case 1 and 2.

Third, the comparisons of the probabilistic damage analysis under uncertain parameters on different points are provided by selecting three feature nodes (the locations are shown in Fig. 3(b) ), and only Case 3 is investigated with the results shown in Fig. 9(b), From Fig. 9(b), Node 1 is the location where the damage is largest and the distribution of $f(d)$ varies in a smaller range (the standard deviation is $\hat{\sigma}=0.030$ ). Comparing Nodes 1,2 and 3, it can be found that the location with less damage is more sensitive to the uncertain parameters, and the sensitivity increases as the damage level increases such that the standard deviation $\hat{\sigma}$ of the damage function $f(d)$ increases from 0.03 to 0.087 when the mean value $\hat{\mu}$ of $f(d)$ increases from 0.066 to 0.41 .

Finally, the levels of uncertainty for parameters are also investigated and the results are shown in Figs. 9(c) and 9(d), Cases 4-6 are tested to illustrate the influence of the standard deviation $\hat{\sigma}$ when the mean value $\hat{\mu}$ is the same. It can be seen in Fig. 9(c) that when the mean value of the uncertain parameters is constant, the mean value of the damage function $f(d)$ changes slightly, and the standard deviation of $f(d)$ increases as the standard deviation of the uncertain input parameters increases. The influence of the mean value of the input parameters is also investigated in Fig. 9(d). It can be seen that the change of the mean value of the input parameters may affect the distribution of the damage function $f(d)$ such that a larger mean value of the input parameters leads to more serious damage and the distribution of the damage function will be more concentrated. 


\subsection{Radial dilatation of an idealized artery}

The second example is damage analysis for radial dilatation of an idealized artery. The two-dimensional geometry and FEM mesh shown in Fig. 10 were previously established by Badel et al. 44] according to histological pictures of epicardial coronary arteries from VilesGonzalez et al. [45. The artery is assumed to consist of a single medial layer containing an atherosclerotic plaque, and the balloon used for the angioplasty is modeled as a thin circular structure whose diameter increases during the angioplasty process. In this example, the damage is assumed to only occur in the artery. Based on the experiment data for human thoracic and abdominal aortas in Weisbecker et al. [46], the shear modulus of the artery is assumed obeys a beta distribution $\mu_{\mathrm{e}} \sim B(2.98,8.72)$ within the interval $[15,55] \mathrm{kPa}$. The internal length scales $c_{\mathrm{d}}$ is assumed to obey a beta distribution $c_{\mathrm{d}} \sim B(4,4)$ within the interval $[0.005,0.015] \mathrm{kPa}^{-1} \cdot \mathrm{mm}^{2}$.

In this simulation, some simplifications are assumed, i.e., the influence of the residual stresses, smooth muscle, lipid pool, and the image-based layer-specific structure of the arteries are not considered, and an idealized geometric shape is used. The only boundary conditions to be assigned are the nodal displacements of the balloon. A radial displacement is imposed on each node from its initial position, $D_{i}=1 \mathrm{~mm}$, to give a final deformed diameter, $D_{f}=2 \mathrm{~mm}$, where the center of the balloon is fixed. In the following, we use an inflation progress parameter $\lambda=\left(D-D_{i}\right) /\left(D_{f}-D_{i}\right) \times 100 \%$, where $D$ is the current diameter of the balloon, to denote the inflation progress. Note that all the degrees of freedom of the balloon are prescribed as displacement boundary conditions, and the artery is inflated by the contact between the balloon and artery. The contact problems (balloon and plaque, balloon and artery, plaque and artery) are assumed to be frictionless following Badel et al. 44 and are modeled as 'Hard contact' in Abaqus. The material parameters used in this example shown in Table 5 are according to Badel et al. [44] and Weisbecker et al. [46].

\subsubsection{Verification of the surrogate model}

Similar with the previous example, three different cases, including (1) $\mu_{\mathrm{e}}$ is uncertain with a constant $c_{\mathrm{d}},(2) c_{\mathrm{d}}$ is uncertain with a constant $\mu_{\mathrm{e}}$ and (3) both $\mu_{\mathrm{e}}$ and $c_{\mathrm{d}}$ are uncertain, are tested and the distributions of the uncertain input parameters are shown in Table6. The 5 -order Legendre polynomial surrogate model is used and the SGCM is set by setting $k=5$. To check the accuracy of surrogate model, the damage function $f(d)$ is calculated by the surrogate model and the direct FEM for 3 cases, and the results shown in Fig. 11 illustrate the good performance of the surrogate model. The contours of the damage function $f(d)$ calculated by the surrogate model with SGCM and FEM for different shear modulus and the internal length scales when $\lambda=100 \%$ in Fig. 12 demonstrate the good performance of the surrogate model once again.

\subsubsection{Probability analysis}

First, the computational cost of probability analysis for using the Monte Carlo method with the direct FEM and the Monte Carlo method with the surrogate model are investigated in Table 7. The results again demonstrate that the surrogate model can effectively reduce the computational cost with good accuracy, as shown in Fig. 11 . 
Second, the individual and combined impacts of the uncertain parameters on the damage are tested, and the PDFs of the damage function $f(d)$ for three cases in Table 6 at different nodes (the locations are shown in Fig. 10 are shown in Fig. 13. Note that Node 1 is the location where the damage is the largest in the whole domain and it is selected through a previous simulation. For these three cases, the distribution of the damage function at Node 1 is closer to 0, which means larger damage. Comparing the results of Case 1 for these three nodes, the same results that a larger influence of the shear modulus on the location with less damage can be observed, which the standard deviation $\hat{\sigma}$ of the damage function $f(d)$ increase form 0.009 to 0.054. By comparing the results of $f(d)$ for Cases 1 and 3 for these three nodes, only a visible difference at Node 1 can be found and $f(d)$ varies in a larger range at Node 1 for Case $2(\hat{\sigma}=0.019)$, which illustrates that value of the internal length scales $c_{\mathrm{d}}$ has a larger impact on the location with larger damage.

Moreover, three different ranges of the internal length scales $c_{\mathrm{d}}$, that $c_{\mathrm{d}} \in[0.002,0.018]$, $c_{\mathrm{d}} \in[0.005,0.015]$ and $c_{\mathrm{d}} \in[0.008,0.012]$, are tested to investigate the influence of the nonlocal damage parameter when $\lambda=100 \%$. In this situation, the shear modulus of the artery remains a constant such that $\mu_{\mathrm{e}}=28.97 \mathrm{kPa}$, and the PDF of $f(d)$ at Node 1 is shown in Fig. 14(a), The value of the damage function $f(d)$ varies in the range of $f(d) \in$ $[0.20,0.25]$ when $c_{\mathrm{d}} \in[0.008,0.012]$, and the interval of the damage for $c_{\mathrm{d}} \in[0.002,0.018]$ is $f(d) \in[0.10,0.31]$, which is 3 times larger than the width when $c_{\mathrm{d}} \in[0.008,0.012]$. Although the mean values of the PDFs of the damage function $f(d)$ are close, the standard derivation increases from 0.011 to 0.029 . When the range of $c_{\mathrm{d}}$ is larger, the damage function will be distributed over a larger interval. To evaluate the risk of an artery rupture, the PoF is calculated as introduced in Section 2.6. In this example, the maximum damage threshold is set to $f(d)^{\max }=0.5$ in Eq. (32), which means that an artery rupture could occur when $f(d) \leq f(d)^{\max }$. Since Node 2 is the location where the damage is the largest, the PoF of the artery for Case 3 is calculated by integrating the PDF of the damage function $f(d)$ at Node 2 from $f(d)=0$ to $f(d)=0.5$ through Eq. (33). When $f(d)^{\max }=0.5$ and $\lambda=100 \%$, the PoF of the artery is 1.0 , which means that when $\lambda=100 \%$, there is a $100 \%$ chance of a rupture during the treatment.

As the size of the inflation of the balloon is the critical indicator in clinical treatment [47], the propagation of the uncertainty of the damage function $f(d)$ with uncertain parameters under different inflation sizes is calculated. Four different inflation progress parameters $\lambda$ are used to study the influence of $\lambda$ on the PoF of the artery, and the results are shown in Fig. 14(b). It can be seen that the PoF of $f(d)$ is directly influenced by the inflation progress parameter $\lambda$. For instance, the PoF are 0.03 and 0.91 for $\lambda=90 \%$ and $\lambda=95 \%$, respectively. Obviously, the PoF of the artery increases as the balloon inflation size increases.

\section{Discussion and conclusions}

Due to the widely-existing uncertainty in damage analysis for soft biological tissues, the sensitivity analysis of nonlocal damage for soft biological tissues is an essential step in model development and application. To investigate the influence of the uncertain input parameters on the mechanical environment of the soft biological tissues, a numerical analysis 
is conducted by combining the gradient-enhanced damage model [10, 11] and the surrogate model-based probability analysis method [33].

The first advantage of the presented model is that it can assess nonlocal damage with uncertain material parameters, including internal length scales. In our previous works [12, 13], it was proved that the internal length scales have obvious effects on the localization of damage, e.g., a larger internal length scales lead to a larger lower level of damage and activated zone. In this paper, the influence of uncertain internal length scales can be analyzed by the proposed model. Therefore, it is possible to provide probabilistic results of the levels of damage, stress and deformation for soft tissue if the internal length scales are uncertain variables obeying a probability distribution. Moreover, the proposed model can consider the combined effects of different uncertain material parameters. For instance, both the shear modulus and an internal length scales parameter can be set as uncertain variables at the same time. This will be useful for dealing with the cases in which more uncertain parameters exist together.

The other advantage is that this paper's method provides a new approach for the uncertain damage analysis of soft biological tissues based on the surrogate model. Although the Monte Carlo method is an accurate and robust method for uncertainty analysis, the huge computational cost is a key challenge for its application to damage analyses since the nonlinear problem has to be repeatedly solved by the FEM [48, 49]. Due to the huge computational cost, the Monte Carlo method is commonly introduced as a referenced approach, but it is rarely used in the practical engineering. In this paper, the surrogate model based on the Legendre polynomial series was adopted to approximate the results of the FEM. When training of the surrogate model, the SGCM is employed to accelerate the procedure. First, it is demonstrated that the surrogate model has good accuracy compared with direct FEM solutions. For example, the maximum error is less than $1 \%$ compared with the results (Cauchy stress and the damage function) obtained from the surrogate model and the FEM at some feature nodes in the open-hole plate. Second, the surrogate model significantly improved the computational efficiency of the Monte Carlo method. For example, in the open-hole plate problem, the computational cost for the Monte Carlo method with the direct FEM was about 40 days, but the time for the surrogate model with the SGCM including training the surrogate model and probability analysis was only $0.63 \mathrm{~h}$ with a 286 elements mesh.

The proposed approach was applied to the simulation of the radial dilatation of an idealized artery by considering the uncertain shear modulus and internal length scales. Although some simplifications are assumed, some interesting results are found: (1) the spatial positions and level of damage could have significant impact on the uncertainty propagation of damage, for instance, the mean value and standard deviation are obviously different at different locations (Node 1, Node 2 and Node 3) in Fig. 13: (2) different parameters could also have obviously different impact on the uncertainty propagation of damage. For instance, if only the uncertain shear modulus is considered (Case 1), a smaller standard deviation of damage is produced at Node 1 with larger damage compared with Node 3, while the internal length scales has an opposite trend with a larger standard deviation at Node 1 compared with Node 3 in Case 2; (3) although the PoF is increased with the increase of inflation size as expected, the relation between uncertain parameter and resulted PoF could still provide 
a potential helpful approach for both directly predicting the PoF in clinical operation and inversely choosing the inflation size under a prescribed PoF.

The main limitation in this paper is that only 2D models and an idealized geometric model was considered. The development of a 3D patient-specific model is currently under progress in order to address more realistic applications. Meanwhile, some important aspects, such as the influence of the residual stresses, the smooth muscle activation, the lipid pool should also be considered. Besides, some uncertainty analyses about the geometry and the material parameters of the plaque, which is important in predicting the rupture risk of the artery, should be conducted, and the spatial uncertainty should also be considered as arteries are usually heterogeneous materials [50, 51]. Moreover, there is still a pressing need to develop further experiments to identify the distribution of shear moduli, the internal length scales and other unknown material parameters. Furthermore, the use of UEL presents some limitations such as, for instance, the definition of slave surfaces in contact analyses.

In summary, we have coupled a nonlocal damage model and a surrogate model to investigate the influence of uncertainty on damage in soft tissues. The Legendre polynomial surrogate model with the SGCM was adopted in this paper to speed up the computation efficiency. The evolution of the PDF of the damage function $f(d)$ was illustrated through two examples where the input parameters were assumed to be uncertain, and the method's clinical application to balloon angioplasty was also addressed. Future work will consist of applying the proposed model to practical problems by collecting clinical data.

\section{Acknowledgments}

The research leading to this paper was funded by the NSFC Grant [12072063], ERC2014-CoG-BIOLOCHANICS [647067], grants from the State Key Laboratory of Structural Analysis for Industrial Equipment [S18402, GZ19105], the Liaoning Provincial Natural Science Foundation [2020-MS-110] and the Visiting Scholar Foundation of Key Laboratory of Biorheological Science and Technology (Chongqing University) from Ministry of Education [CQKLBST-2020-002].

\section{References}

[1] Mousavi SJ, Farzaneh S, Avril S. Computational predictions of damage propagation preceding dissection of ascending thoracic aortic aneurysms. International journal for numerical methods in biomedical engineering 2018; 34(4): e2944.

[2] Fleischmann W, Strecker W, Bombelli M, Kinzl L. Vacuum sealing as treatment of soft tissue damage in open fractures. Der Unfallchirurg 1993; 96(9): 488-492.

[3] Kloner R, Ellis S, Lange R, Braunwald E. Studies of experimental coronary artery reperfusion. Effects on infarct size, myocardial function, biochemistry, ultrastructure and microvascular damage.. Circulation 1983; 68(2 Pt 2): I8-15.

[4] Holzapfel GA, Fereidoonnezhad B. Modeling of damage in soft biological tissues. In: Elsevier. 2017 (pp. 101-123).

[5] Trabelsi O, Davis FM, Rodriguez-Matas JF, Duprey A, Avril S. Patient specific stress and rupture analysis of ascending thoracic aneurysms. Journal of biomechanics 2015; 48(10): 1836-1843.

[6] Svensson R, Mulder H, Kovanen V, Magnusson SP. Fracture Mechanics of Collagen Fibrils: Influence of Natural Cross-Links. Biophysical Journal 2013; 104(11): 2476-2484. 
[7] Holzapfel GA, Stadler M, Gasser TC. Changes in the mechanical environment of stenotic arteries during interaction with stents: computational assessment of parametric stent designs. Journal of biomechanical engineering 2005; 127(1): 166-180.

[8] Gasser TC. Damage in vascular tissues and its modeling. In: Springer. 2017 (pp. 85-118).

[9] Li , Wenguang . Damage Models for Soft Tissues: A Survey. Journal of Medical \& Biological Engineering 2016; 36(3): 285-307.

[10] Dimitrijevic B, Hackl K. A method for gradient enhancement of continuum damage models. Technische Mechanik 2008; 28(1): 43-52.

[11] Dimitrijevic B, Hackl K. A regularization framework for damage-plasticity models via gradient enhancement of the free energy. International Journal for Numerical Methods in Biomedical Engineering 2011; 27(8): 1199-1210.

[12] He Y, Zuo D, Hackl K, Yang H, Mousavi SJ, Avril S. Gradient-enhanced continuum models of healing in damaged soft tissues. Biomechanics and Modeling in Mechanobiology 2019; 18: 1-18.

[13] Zuo D, Avril S, Yang H, Mousavi SJ, Hackl K, He Y. Three-dimensional numerical simulation of soft-tissue wound healing using constrained-mixture anisotropic hyperelasticity and gradient-enhanced damage mechanics. Journal of the Royal Society Interface 2020; 17(162): 20190708.

[14] Paul H, Hale JS, Stéphane C, Bordas SPA. Quantifying the uncertainty in a hyperelastic soft tissue model with stochastic parameters. Applied Mathematical Modelling 2018; 62: 86-102.

[15] Gasser TC, Grytsan A. Biomechanical modeling the adaptation of soft biological tissue. Current Opinion in Biomedical Engineering 2017: S2468451117300272.

[16] Laz PJ, Browne M. A review of probabilistic analysis in orthopaedic biomechanics. Proceedings of the Institution of Mechanical Engineers, Part H: Journal of Engineering in Medicine 2010; 224(8): 927-943.

[17] Rodríguez JF, Cacho F, Bea JA, Doblaré M. A stochastic-structurally based three dimensional finitestrain damage model for fibrous soft tissue. Journal of the Mechanics and Physics of Solids 2006; 54(4): 864-886.

[18] Schmidt T, Balzani D, Holzapfel GA. Statistical approach for a continuum description of damage evolution in soft collagenous tissues. Computer Methods in Applied Mechanics and Engineering 2014; 278: 41-61.

[19] Balzani D, Schmidt T, Ortiz M. Method for the quantification of rupture probability in soft collagenous tissues. International journal for numerical methods in biomedical engineering 2017; 33(1): e02781.

[20] Lee T, Bilionis I, Tepole AB. Propagation of uncertainty in the mechanical and biological response of growing tissues using multi-fidelity Gaussian process regression. Computer Methods in Applied Mechanics and Engineering 2020; 359: 112724.

[21] Fishman G. Monte Carlo: concepts, algorithms, and applications. Springer Science \& Business Media 2013.

[22] Ong YS, Nair PB, Keane AJ. Evolutionary optimization of computationally expensive problems via surrogate modeling. AIAA journal 2003; 41(4): 687-696.

[23] Gorissen D, Couckuyt I, Demeester P, Dhaene T, Crombecq K. A surrogate modeling and adaptive sampling toolbox for computer based design. Journal of Machine Learning Research 2010; 11(Jul): 2051-2055.

[24] Díaz-Manríquez A, Toscano G, Barron-Zambrano JH, Tello-Leal E. A review of surrogate assisted multiobjective evolutionary algorithms. Computational intelligence and neuroscience 2016; 2016.

[25] Razavi S. Review of surrogate modeling in water resources. Water Resources Research 2012.

[26] Asher MJ, Croke BFW, Jakeman AJ, Peeters LJM. A review of surrogate models and their application to groundwater modeling. Water Resources Research 2015; 51(8): 5957-5973.

[27] Zhang W. A Review of Surrogate Models: 7-17; Singapore: Springer Singapore . 2020

[28] Yondo R, Bobrowski K, Andrés E, Valero E. A Review of Surrogate Modeling Techniques for Aerodynamic Analysis and Optimization: Current Limitations and Future Challenges in Industry: 19-33; Cham: Springer International Publishing . 2019

[29] Myers RH, Montgomery DC, Anderson-Cook CM. Response surface methodology: process and product optimization using designed experiments. John Wiley \& Sons . 2016. 
[30] Hardy RL. Multiquadric equations of topography and other irregular surfaces. Journal of geophysical research 1971; 76(8): 1905-1915.

[31] Sacks J, Welch WJ, Mitchell TJ, Wynn HP. Design and analysis of computer experiments. Statistical science 1989: 409-423.

[32] Wang C, Qiu Z, Yang Y. Collocation methods for uncertain heat convection-diffusion problem with interval input parameters. International Journal of Thermal Sciences 2016; 107: 230-236.

[33] Wang H, Xiang S. On the convergence rates of Legendre approximation. Mathematics of Computation 2012; 81(278): 861-877.

[34] Waffenschmidt T, Polindara C, Menzel A, Blanco S. A gradient-enhanced large-deformation continuum damage model for fibre-reinforced materials. Computer Methods in Applied Mechanics and Engineering 2014; 268: 801-842.

[35] Holzapfel GA, Gasser TC, Ogden RW. A New Constitutive Framework for Arterial Wall Mechanics and a Comparative Study of Material Models. Journal of Elasticity 2000; 61(1-3): 1-48.

[36] Gasser TC, Ogden RW, Holzapfel GA. Hyperelastic modelling of arterial layers with distributed collagen fibre orientations. Journal of the Royal Society Interface 2006; 3(6).

[37] Simo JC, Ju J. Strain-and stress-based continuum damage models-I. Formulation. International journal of solids and structures 1987; 23(7): 821-840.

[38] Raghavan ML, Webster MW, Vorp DA. Ex vivo biomechanical behavior of abdominal aortic aneurysm: assessment using a new mathematical model. Annals of Biomedical Engineering 1996; 24(5): 573-582.

[39] Xiu D, Karniadakis GE. Modeling uncertainty in flow simulations via generalized polynomial chaos. Journal of computational physics 2003; 187(1): 137-167.

[40] Smolyak S. Quadrature and Interpolation Formulas for Tensor Products of Certain Classes of Functions. Doklady Akademii Nauk Sssr 1963; 4(5): 240-243.

[41] Johnson NL, Kotz S, Balakrishnan N. Continuous univariate distributions. Hougton Mifflin . 1970.

[42] Biehler J, Wall W. The impact of personalized probabilistic wall thickness models on peak wall stress in abdominal aortic aneurysms. International journal for numerical methods in biomedical engineering 2018; 34(2): e2922.

[43] Wu D, Gao W, Wang C, Tangaramvong S, Tin-Loi F. Robust fuzzy structural safety assessment using mathematical programming approach. Fuzzy Sets $\mathcal{E}$ Systems 2016; 293(jun.15): 30-49.

[44] Badel P, Avril S, Sutton MA, Lessner SM. Numerical simulation of arterial dissection during balloon angioplasty of atherosclerotic coronary arteries. Journal of biomechanics 2014; 47(4): 878-889.

[45] Viles-Gonzalez JF, Castro Miranda dR, Scanavacca M, Sosa E, d'Avila A. Acute and chronic effects of epicardial radiofrequency applications delivered on epicardial coronary arteries. Circulation: Arrhythmia and Electrophysiology 2011; 4(4): 526-531.

[46] Weisbecker H, Pierce DM, Regitnig P, Holzapfel GA. Layer-specific damage experiments and modeling of human thoracic and abdominal aortas with non-atherosclerotic intimal thickening. Journal of the mechanical behavior of biomedical materials 2012; 12: 93-106.

[47] Tenaglia AN, others. Intravascular ultrasound and balloon percutaneous transluminal coronary angioplasty. Cardiology clinics 1997; 15(1): 31-38.

[48] Neumaier A. Interval methods for systems of equations. 37. Cambridge university press . 1990.

[49] Ran C, Yang H, Zhang G. A gradient based algorithm to solve inverse plane bimodular problems of identification. Journal of Computational Physics 2018; 355: 78-94.

[50] Bersi MR, Bellini C, Di Achille P, Humphrey JD, Genovese K, Avril S. Novel Methodology for Characterizing Regional Variations in the Material Properties of Murine Aortas. Journal of Biomechanical Engineering 2016; 138(7). 071005.

[51] Bersi MR, Santamaría VAA, Marback K, Achille PD, Avril S. Multimodality Imaging-Based Characterization of Regional Material Properties in a Murine Model of Aortic Dissection. Scientific Reports $2020 ; 10(1)$. 
List of Tables

577

$1 \quad$ Geometry, hyperelastic and damage parameters used in the open-hole plate.

2 The uncertain input parameters for different cases. . . . . . . . . . . . . 20

3 Comparison of the results obtained by the FEM and surrogate model with the SGCM when $\lambda_{x}=0.1$ for Case 1. . . . . . . . . . . . . . . . 21

580

581

582

\section{4} The comparison of the computational cost between using the Monte Carlo

583 method with the direct FEM and the Monte Carlo method with the surrogate

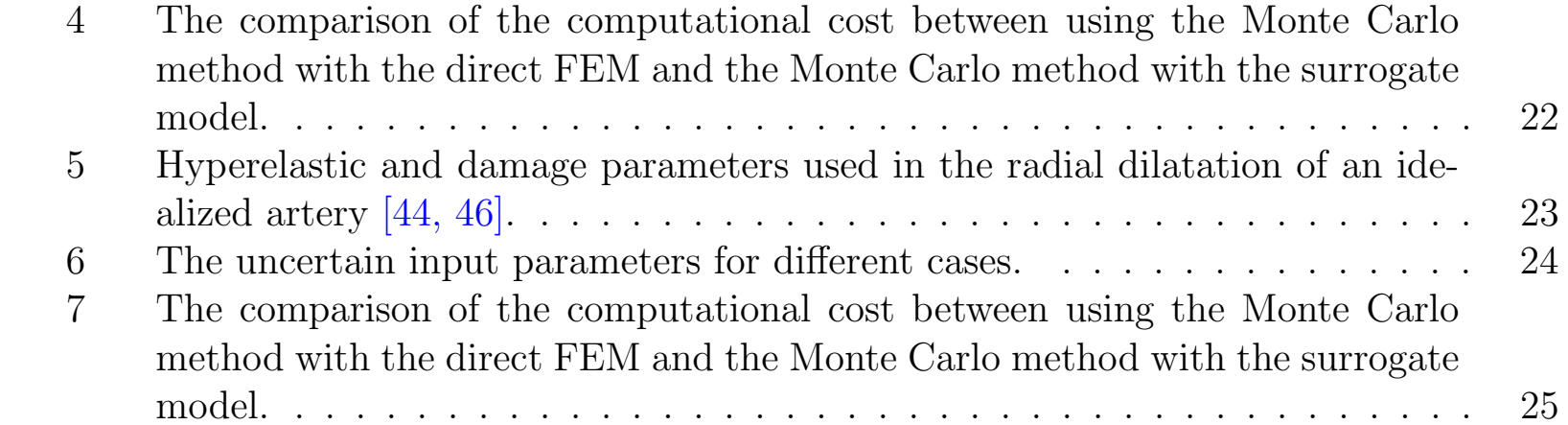


Table 1: Geometry, hyperelastic and damage parameters used in the open-hole plate.

\begin{tabular}{lllll}
\hline Type & Description & Symbol & Values & Units \\
\hline \multirow{3}{*}{ Geometric } & Height & $\mathrm{H}$ & 200 & $\mathrm{~mm}$ \\
& Width & $\mathrm{W}$ & 200 & $\mathrm{~mm}$ \\
& Radius & $\mathrm{R}$ & 50 & $\mathrm{~mm}$ \\
\hline \multirow{2}{*}{ Hyperelastic } & Shear modulus & $\mu_{0}$ & {$[0.5,1.0,1.5]$} & $\mathrm{MPa}$ \\
& Bulk modulus & $\kappa_{0}$ & {$[5,10,15]$} & $\mathrm{MPa}$ \\
\hline \multirow{5}{*}{ Damage } & Damage threshold & $r_{1}$ & 0.001 & $\mathrm{MPa}$ \\
& Internal length scales & $c_{\mathrm{d}}$ & {$[0.5,1,5]$} & $\mathrm{MPa}^{-1} \cdot \mathrm{mm}^{2}$ \\
& Penalty parameter & $\beta_{\mathrm{d}}$ & 0.005 & $\mathrm{MPa}^{-1}$ \\
& (Non)local switch & $\gamma_{\mathrm{d}}$ & 1 & - \\
\hline
\end{tabular}


Table 2: The uncertain input parameters for different cases.

\begin{tabular}{|c|c|c|c|c|c|c|}
\hline \multirow{2}{*}{ Case } & \multicolumn{3}{|c|}{$\mu_{\mathrm{e}}(\mathrm{MPa})$} & \multicolumn{3}{|c|}{$c_{\mathrm{d}}\left(\mathrm{MPa}^{-1} \cdot \mathrm{mm}^{2}\right)$} \\
\hline & Distribution & Mean & Standard Deviation & Distribution & Mean & Standard Deviation \\
\hline 1 & $B(4,4)$ & 0.1 & 0.01 & - & 1 & - \\
\hline 2 & - & 0.1 & - & $B(4,4)$ & 1 & 0.17 \\
\hline 3 & $B(4,4)$ & 0.1 & 0.01 & $B(4,4)$ & 1 & 0.17 \\
\hline 4 & $B(2,2)$ & 0.1 & 0.013 & $B(2,2)$ & 1 & 0.22 \\
\hline 5 & $B(8,8)$ & 0.1 & 0.0073 & $B(8,8)$ & 1 & 0.12 \\
\hline 6 & $B(4,2)$ & 0.11 & 0.0107 & $B(4,2)$ & 1.17 & 0.18 \\
\hline 7 & $B(2,4)$ & 0.09 & 0.0107 & $B(2,4)$ & 0.83 & 0.18 \\
\hline
\end{tabular}


Table 3: Comparison of the results obtained by the FEM and surrogate model with the SGCM when $\lambda_{x}=0.1$ for Case 1.

\begin{tabular}{|c|c|c|c|c|c|c|c|}
\hline \multirow{2}{*}{ Node } & \multirow[b]{2}{*}{$\mu_{\mathrm{e}}$} & \multicolumn{2}{|c|}{ FEM } & \multicolumn{4}{|c|}{ SGCM } \\
\hline & & $f(d)$ & $\sigma_{x}$ & $f(d)$ & error & $\sigma_{x}$ & error \\
\hline \multirow{2}{*}{1} & 0.08 & 0.142469 & 0.028497 & 0.141972 & $0.35 \%$ & 0.028479 & $0.06 \%$ \\
\hline & 0.12 & 0.028766 & 0.026306 & 0.028787 & $0.07 \%$ & 0.026207 & $0.38 \%$ \\
\hline \multirow{2}{*}{2} & 0.08 & 0.38338 & 0.031512 & 0.382846 & $0.14 \%$ & 0.031431 & $0.26 \%$ \\
\hline & 0.12 & 0.161063 & 0.031508 & 0.161157 & $0.06 \%$ & 0.03152 & $0.04 \%$ \\
\hline \multirow{2}{*}{3} & 0.08 & 0.607423 & 0.014506 & 0.605692 & $0.28 \%$ & 0.014729 & $0.05 \%$ \\
\hline & 0.12 & 0.293042 & 0.015549 & 0.294017 & $0.33 \%$ & 0.015552 & $0.02 \%$ \\
\hline \multirow{2}{*}{4} & 0.08 & 1 & 0.001851 & 1 & $0.00 \%$ & 0.001855 & $0.23 \%$ \\
\hline & 0.12 & 1 & 0.001331 & 1 & $0.00 \%$ & 0.001342 & $0.80 \%$ \\
\hline \multirow{2}{*}{5} & 0.08 & 1 & -0.00159 & 1 & $0.00 \%$ & -0.00159 & $0.10 \%$ \\
\hline & 0.12 & 1 & -0.00086 & 1 & $0.00 \%$ & -0.00086 & $0.20 \%$ \\
\hline \multirow{2}{*}{6} & 0.08 & 1 & 0.022613 & 1 & $0.00 \%$ & 0.022606 & $0.03 \%$ \\
\hline & 0.12 & 1 & 0.021469 & 1 & $0.00 \%$ & 0.02149 & $0.10 \%$ \\
\hline
\end{tabular}


Table 4: The comparison of the computational cost between using the Monte Carlo method with the direct FEM and the Monte Carlo method with the surrogate model.

\begin{tabular}{cccccc}
\hline & $\begin{array}{c}\text { Monte Carlo method with } \\
\text { the direct FEM } \\
\left(\text { Estimated, } t_{1}\right)\end{array}$ & \multicolumn{3}{c}{ Monte Carlo method with the surrogate model } \\
\cline { 3 - 5 } & 14.70 days & $825 \mathrm{sec}$ & $11.82 \mathrm{sec}$ & $836.82 \mathrm{sec}$ & $0.06 \%$ \\
Surrogate model & $\begin{array}{c}\text { Probability } \\
\text { analysis }\end{array}$ & $\begin{array}{c}\text { Total time } \\
\left(t_{2}\right)\end{array}$ & $t_{1}$ \\
\hline 79 elements & 40.05 days & $2249 \mathrm{sec}$ & $36.28 \mathrm{sec}$ & $2285.28 \mathrm{sec}$ & $0.07 \%$ \\
796 elements & 115.27 days & $6474 \mathrm{sec}$ & $98.73 \mathrm{sec}$ & $6572.73 \mathrm{sec}$ & $0.07 \%$ \\
\hline
\end{tabular}


Table 5: Hyperelastic and damage parameters used in the radial dilatation of an idealized artery [44, 46].

\begin{tabular}{|c|c|c|c|c|c|}
\hline Туре & Part & Description & Symbol & Values & Units \\
\hline \multirow{10}{*}{ Hyperelastic } & \multirow{6}{*}{ Medial layer } & Shear modulus & $\mu_{\mathrm{e}}$ & uncertain & $\mathrm{kPa}$ \\
\hline & & Bulk modulus & $\kappa_{\mathrm{e}}$ & 5 & $\mathrm{MPa}$ \\
\hline & & Material parameter & $k_{1}$ & 5.15 & $\mathrm{MPa}$ \\
\hline & & Material parameter & $k_{2}$ & 8.64 & - \\
\hline & & Dispersion parameter & $\kappa_{0}$ & 0.24 & - \\
\hline & & Fiber orientation angle & $\theta$ & \pm 38.8 & deg \\
\hline & \multirow{2}{*}{ Plaque } & Shear modulus & $\mu_{\mathrm{p}}$ & 20 & $\mathrm{kPa}$ \\
\hline & & Bulk modulus & $\kappa_{\mathrm{p}}$ & 34 & $\mathrm{kPa}$ \\
\hline & \multirow{2}{*}{ Balloon } & Shear modulus & $\mu_{\mathrm{b}}$ & 10 & $\mathrm{kPa}$ \\
\hline & & Bulk modulus & $\kappa_{\mathrm{b}}$ & 10 & $\mathrm{kPa}$ \\
\hline \multirow{4}{*}{ Damage } & \multirow{4}{*}{ Medial layer } & Damage threshold & $r_{1}$ & 1.0 & $\mathrm{kPa}$ \\
\hline & & Internal length scales & $c_{\mathrm{d}}$ & uncertain & $\mathrm{kPa}^{-1} \cdot \mathrm{mm}^{2}$ \\
\hline & & Penalty parameter & $\beta_{\mathrm{d}}$ & 10 & $\mathrm{kPa}^{-1}$ \\
\hline & & (Non-)local switch & $\gamma_{\mathrm{d}}$ & 1.0 & - \\
\hline
\end{tabular}


Table 6: The uncertain input parameters for different cases.

\begin{tabular}{|c|c|c|c|c|c|c|}
\hline \multirow{2}{*}{ Case } & \multicolumn{3}{|c|}{$\mu_{\mathrm{e}}(\mathrm{kPa})$} & \multicolumn{3}{|c|}{$c_{\mathrm{d}}\left(\mathrm{kPa}^{-1} \cdot \mathrm{mm}^{2}\right)$} \\
\hline & Distribution & Mean & Standard Deviation & Distribution & Mean & Standard Deviation \\
\hline 1 & $B(2.98,8.72)$ & 25.58 & 5.03 & \multicolumn{3}{|c|}{0.01 (deterministic) } \\
\hline 2 & \multicolumn{3}{|c|}{25.58 (deterministic) } & $B(4,4)$ & 0.01 & 0.0017 \\
\hline 3 & $B(2.98,8.72)$ & 25.58 & 5.031 & $B(4,4)$ & 0.01 & 0.0017 \\
\hline
\end{tabular}


Table 7: The comparison of the computational cost between using the Monte Carlo method with the direct FEM and the Monte Carlo method with the surrogate model.

\begin{tabular}{ccccr}
\hline \begin{tabular}{c} 
Monte Carlo method with $\begin{array}{c}\text { Carect FEM } \\
\text { the dire } \\
\left(\text { Estimated } t_{1}\right)\end{array}$ \\
\cline { 2 - 4 }
\end{tabular} & $\begin{array}{c}\text { Monte Carlo method with the surrogate model } \\
\text { surrogate model }\end{array}$ & $\begin{array}{c}\text { Probability } \\
\text { analysis }\end{array}$ & $\begin{array}{c}\text { Total time } \\
\left(t_{2}\right)\end{array}$ & $t_{2} / t_{1}$ \\
\hline 230.09 days & $19422 \mathrm{sec}$ & $35.43 \mathrm{sec}$ & $19457.43 \mathrm{sec}$ & $0.09 \%$ \\
\hline
\end{tabular}




\section{List of Figures}

\begin{tabular}{|c|c|}
\hline & experimental stress-strain data of the abdominal aortic aneurysm in Raghavan \\
\hline & et al. [38]. Material parameters are chosen as $\mu_{e}=0.3 \mathrm{MPa}, \kappa_{0}=100 \mathrm{MPa}$, \\
\hline & $k_{1}=0.22 \mathrm{MPa}, k_{2}=1.20, \theta=50 \mathrm{deg}, \varkappa=0.18, r_{1}=0.05 \mathrm{MPa} . \ldots$ \\
\hline 2 & An example for calculating the PoF, in which the damage function is assumed \\
\hline & to obey a beta distribution as $f(d) \sim B(4,4)$ within the interval $(0,1]$ and \\
\hline & the maximum damage is set to $f(d)^{\max }=0.5 . \ldots \ldots \ldots$ \\
\hline 3 & Reference model for an open-hole plate. (a) Geometric, dimension and bound- \\
\hline & ary conditions; and (b) FEM mesh. $\ldots \ldots \ldots \ldots \ldots \ldots$ \\
\hline 4 & The contours of the damage function $f(d)$ for three different shear modulus \\
\hline & 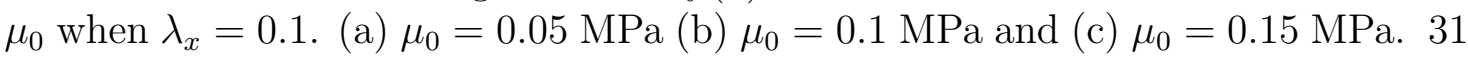 \\
\hline 5 & The average Cauchy stress in $x$-direction $\sigma_{x}$ of the right-hand side for three \\
\hline & different shear modulus $\mu_{0}$ when $\lambda_{x}=0.1 . \ldots \ldots \ldots$. . . . . . . . \\
\hline 6 & The contours of the damage function $f(d)$ for three different internal length \\
\hline & scales $c_{d}$ when $\lambda_{x}=0.1$. (a) $c_{d}=0.5 \mathrm{MPa}^{-1} \cdot \mathrm{mm}^{2}$ (b) $c_{d}=1 \mathrm{MPa}^{-1} \cdot \mathrm{mm}^{2}$ \\
\hline & and $(\mathrm{c}) c_{d}=5 \mathrm{MPa}^{-1} \cdot \mathrm{mm}^{2} . \ldots \ldots \ldots \ldots$ \\
\hline 7 & The average Cauchy stress in $x$-direction $\sigma_{x}$ of the right-hand side for three \\
\hline & different internal length scales $c_{d}$ when $\lambda_{x}=0.1 . \ldots \ldots \ldots \ldots$. . . . . . \\
\hline 8 & The comparison of the surrogate model with the SGCM and FEM. (a) The \\
\hline & Cauchy stress in direction $x$ at Node 1 for Case 2, (b) The damage function \\
\hline & $f(d)$ at Node 1 for Case $2,(\mathrm{c})$ The Cauchy stress in direction $x$ at Node 1 for \\
\hline & Case 3 , and $(\mathrm{d})$ The damage function $f(d)$ at Node 1 for Case $3 . \quad \ldots \ldots$ \\
\hline 9 & PDFs of the damage function $f(d)$ for different situations. (a) PDF of the \\
\hline & $(d)$ at Node 3 for Cases 1-3. (b) PDF of the damage \\
\hline & fferent locations for Case 3. (c) PDF of the damage function \\
\hline & ses 3-5. (d) PDF of the damage function $f(d)$ at Node \\
\hline
\end{tabular}

10 Reference model in the radial dilatation of an idealized artery. (a) Geometry, dimensions and boundary conditions; and (b) FEM mesh. . . . . . . . . . . . 37

11 The comparison of the results obtained by surrogate model with the SGCM and FEM. (a) The damage function $f(d)$ at Node 1 for Case 1, (b) The damage function $f(d)$ at Node 1 for Case 2, and (c) The damage function $f(d)$ at Node 1 for Case 3. . . . . . . . . . . . . . . . . . . . . . . 38

12 The contours of the damage function $f(d)$ obtained by the surrogate model with the SGCM and FEM for different shear modulus and the internal length scales when $\lambda=100 \%$. (a) $\mu_{e}=15 \mathrm{kPa}, c_{d}=0.01 \mathrm{kPa}^{-1} \cdot \mathrm{mm}^{2}$, (b) $\mu_{e}=$ $55 \mathrm{kPa}, c_{d}=0.01 \mathrm{kPa}^{-1} \cdot \mathrm{mm}^{2}$, (c) $\mu_{e}=35 \mathrm{kPa}, c_{d}=0.005 \mathrm{kPa}^{-1} \cdot \mathrm{mm}^{2}$, and (d) $\mu_{e}=35 \mathrm{kPa}, c_{d}=0.015 \mathrm{kPa}^{-1} \cdot \mathrm{mm}^{2} \ldots \ldots \ldots \ldots 39$

13 The PDF of the damage function $f(d)$ for different cases at different locations when $\lambda=100 \%$. . . . . . . . . . . . . . . . . 40 


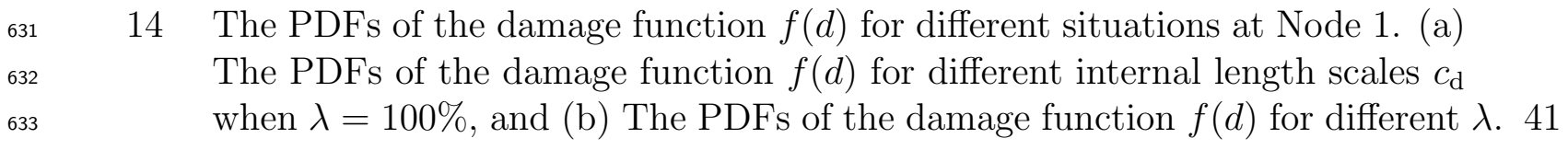




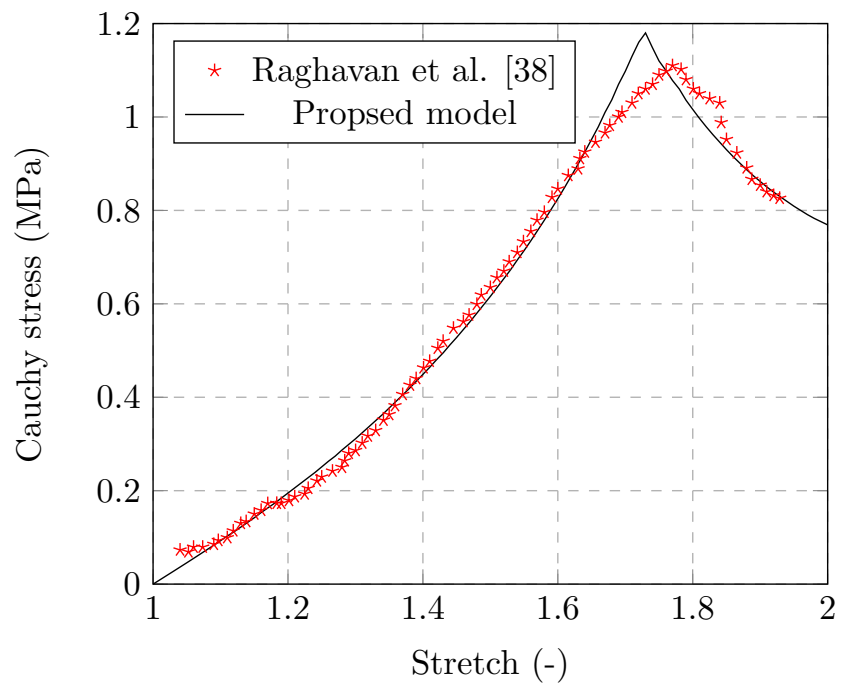

Figure 1: Qualitative simulation based on the damage model used in this paper and the experimental stressstrain data of the abdominal aortic aneurysm in Raghavan et al. 38. Material parameters are chosen as $\mu_{e}=0.3 \mathrm{MPa}, \kappa_{0}=100 \mathrm{MPa}, k_{1}=0.22 \mathrm{MPa}, k_{2}=1.20, \theta=50 \mathrm{deg}, \varkappa=0.18, r_{1}=0.05 \mathrm{MPa}$. 


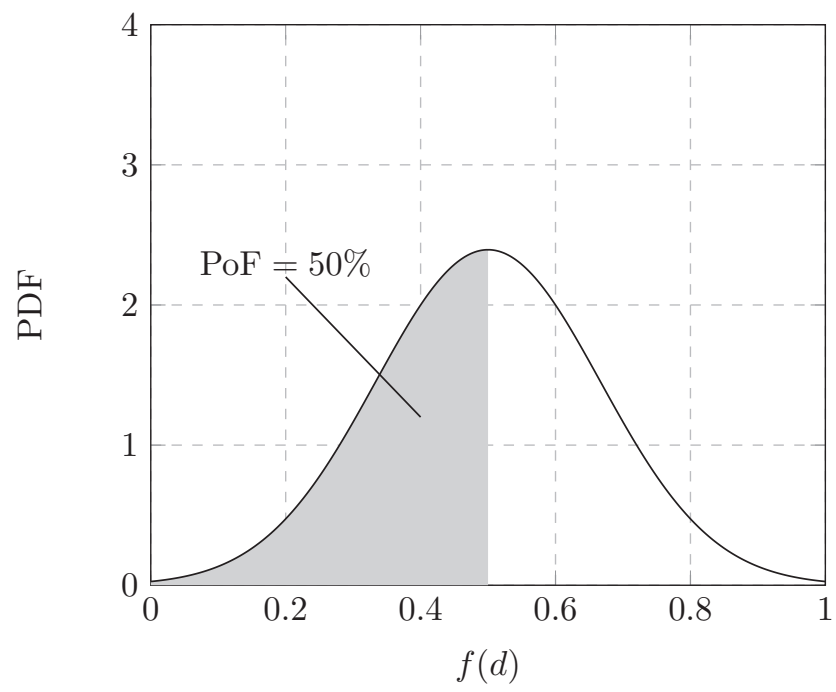

Figure 2: An example for calculating the PoF, in which the damage function is assumed to obey a beta distribution as $f(d) \sim B(4,4)$ within the interval $(0,1]$ and the maximum damage is set to $f(d)^{\max }=0.5$. 


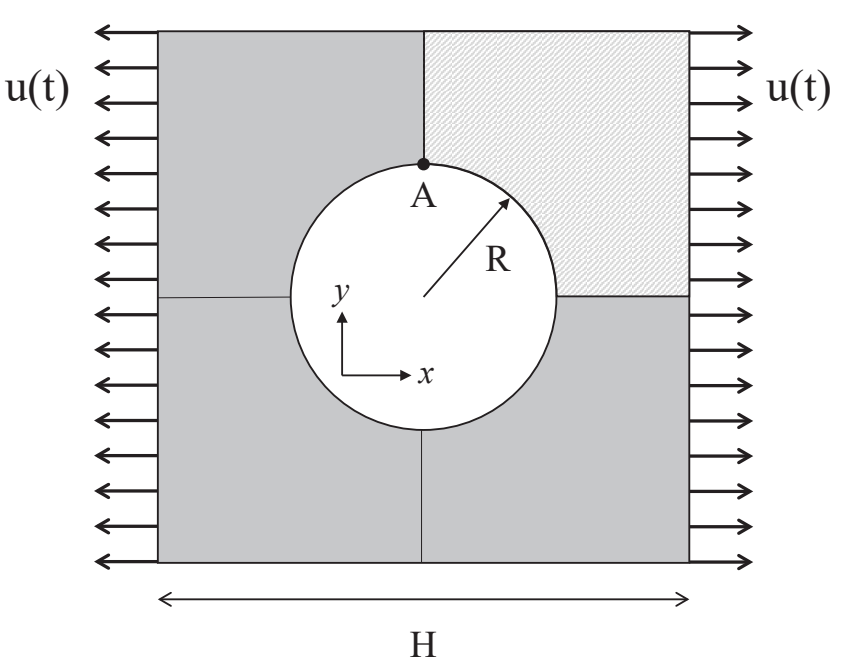

(a)

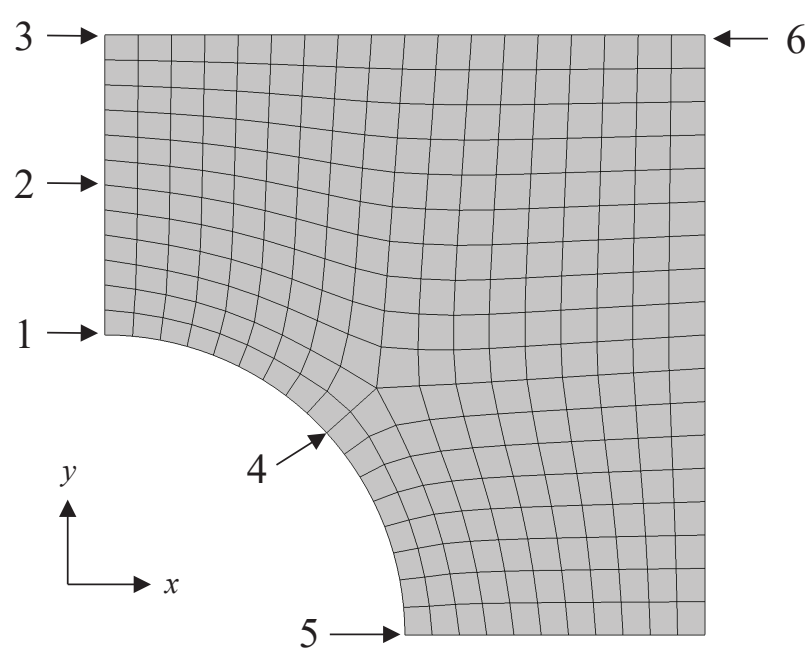

(b)

Figure 3: Reference model for an open-hole plate. (a) Geometric, dimension and boundary conditions; and (b) FEM mesh. 


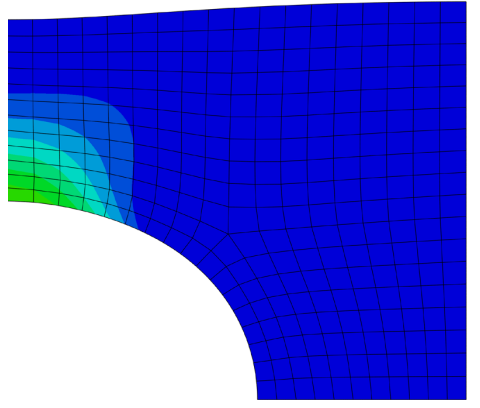

(a)

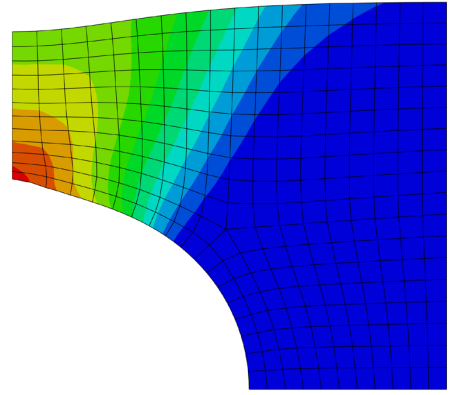

(b)

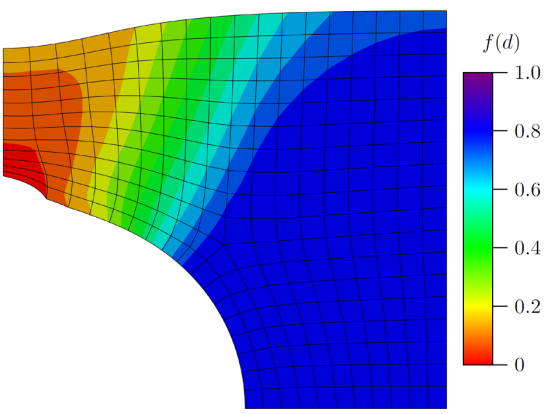

(c)

Figure 4: The contours of the damage function $f(d)$ for three different shear modulus $\mu_{0}$ when $\lambda_{x}=0.1$. (a) $\mu_{0}=0.05 \mathrm{MPa}$ (b) $\mu_{0}=0.1 \mathrm{MPa}$ and (c) $\mu_{0}=0.15 \mathrm{MPa}$. 


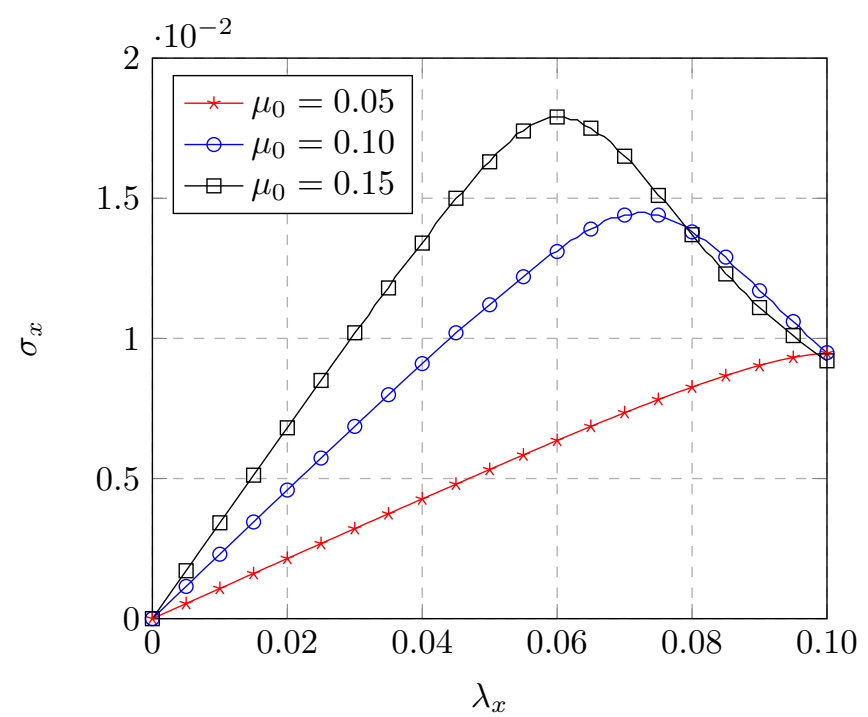

Figure 5: The average Cauchy stress in $x$-direction $\sigma_{x}$ of the right-hand side for three different shear modulus $\mu_{0}$ when $\lambda_{x}=0.1$. 


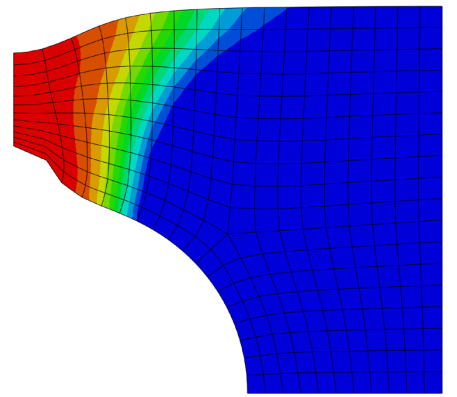

(a)

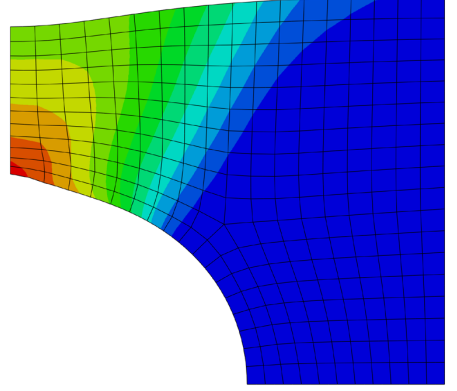

(b)

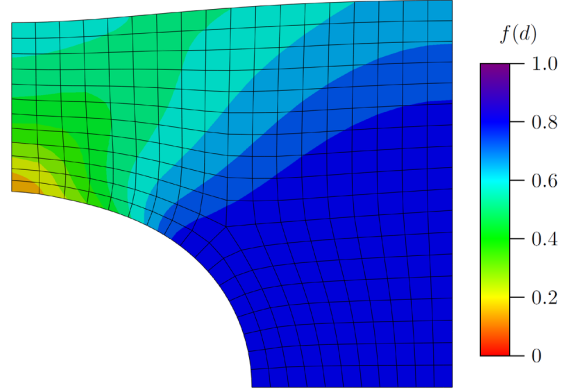

(c)

Figure 6: The contours of the damage function $f(d)$ for three different internal length scales $c_{d}$ when $\lambda_{x}=0.1$. (a) $c_{d}=0.5 \mathrm{MPa}^{-1} \cdot \mathrm{mm}^{2}$ (b) $c_{d}=1 \mathrm{MPa}^{-1} \cdot \mathrm{mm}^{2}$ and (c) $c_{d}=5 \mathrm{MPa}^{-1} \cdot \mathrm{mm}^{2}$. 


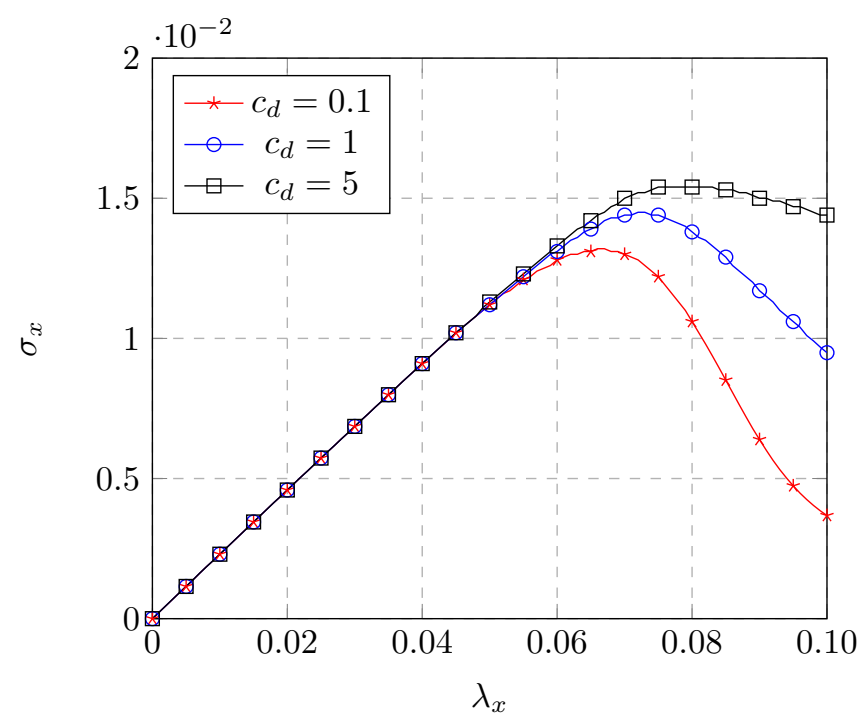

Figure 7: The average Cauchy stress in $x$-direction $\sigma_{x}$ of the right-hand side for three different internal length scales $c_{d}$ when $\lambda_{x}=0.1$. 


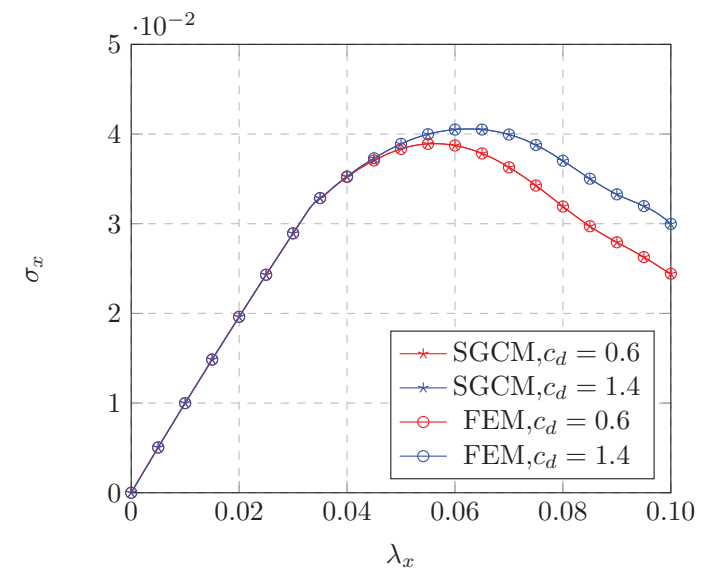

(a)

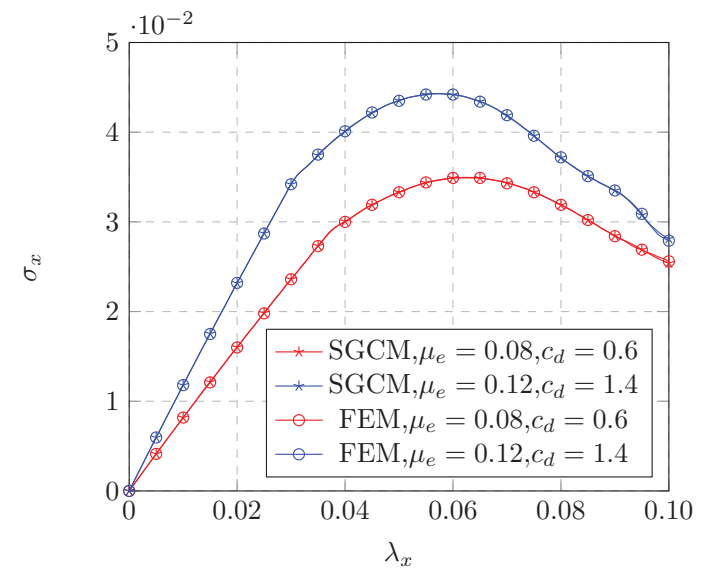

(c)

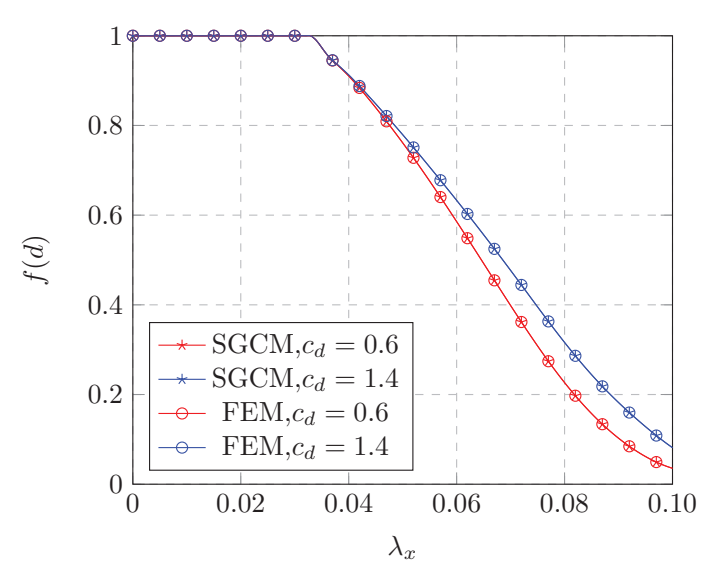

(b)

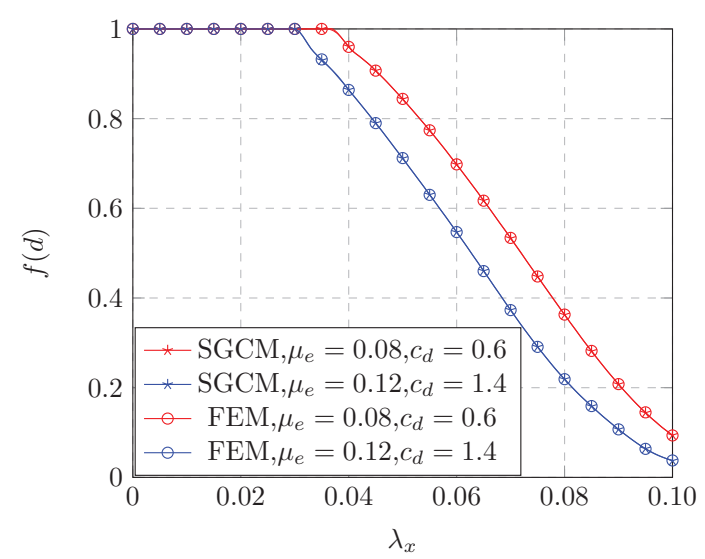

(d)

Figure 8: The comparison of the surrogate model with the SGCM and FEM. (a) The Cauchy stress in direction $x$ at Node 1 for Case 2, (b) The damage function $f(d)$ at Node 1 for Case 2, (c) The Cauchy stress in direction $x$ at Node 1 for Case 3, and (d) The damage function $f(d)$ at Node 1 for Case 3. 


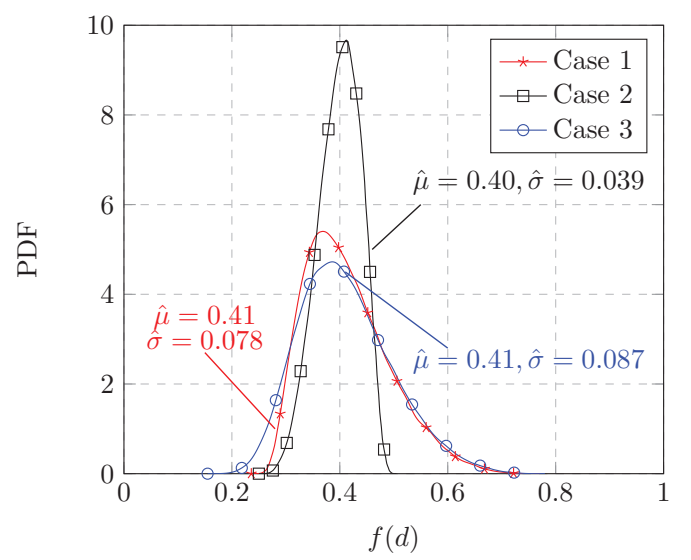

(a)

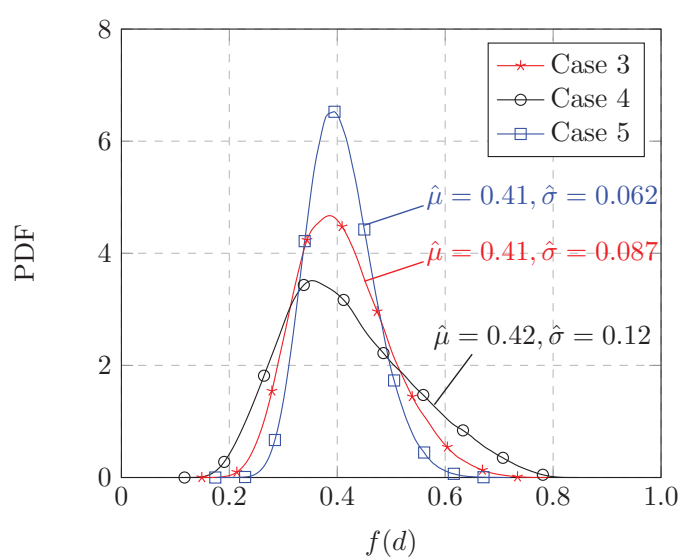

(c)

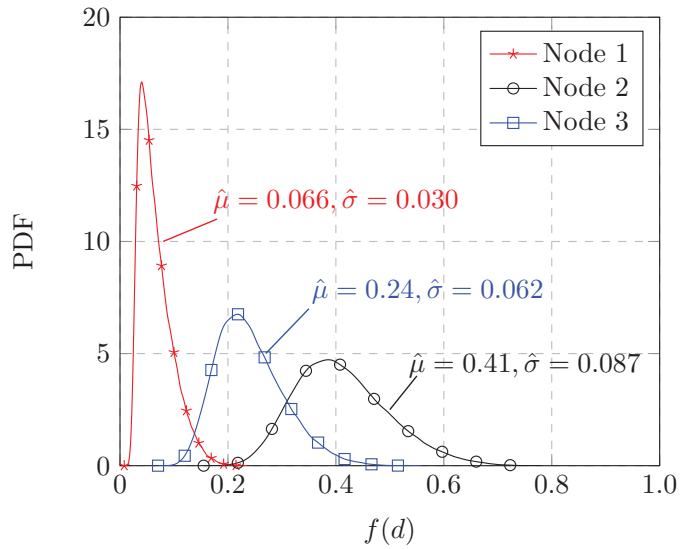

(b)

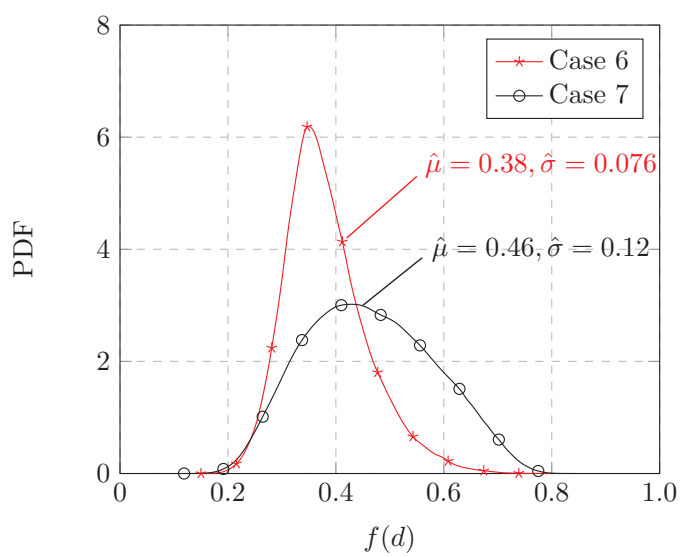

(d)

Figure 9: PDFs of the damage function $f(d)$ for different situations. (a) PDF of the damage function $f(d)$ at Node 3 for Cases 1-3. (b) PDF of the damage function $f(d)$ at different locations for Case 3. (c) PDF of the damage function $f(d)$ at Node 3 for Cases 3-5. (d) PDF of the damage function $f(d)$ at Node 3 for Case 6 and Case 7. 


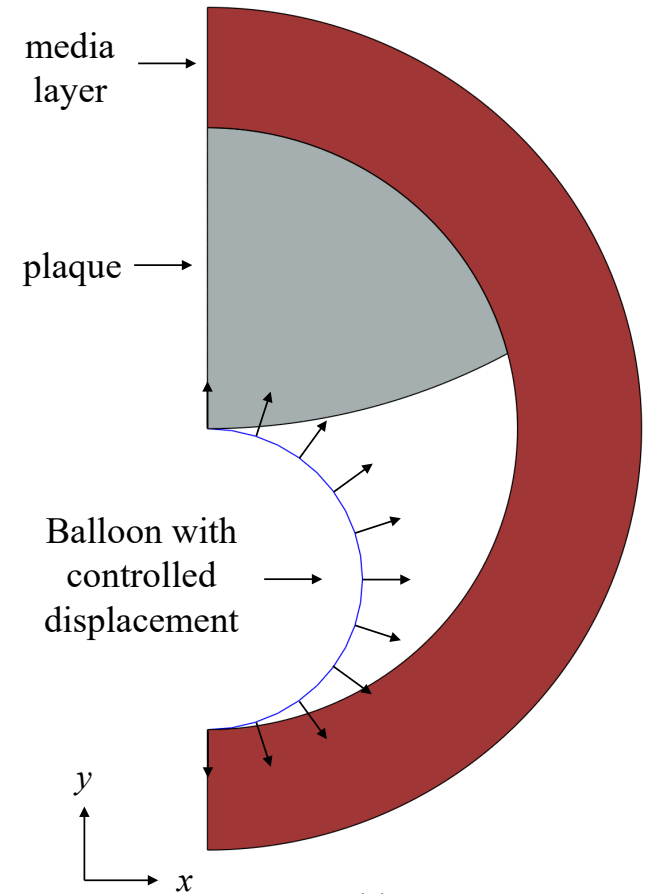

(a)

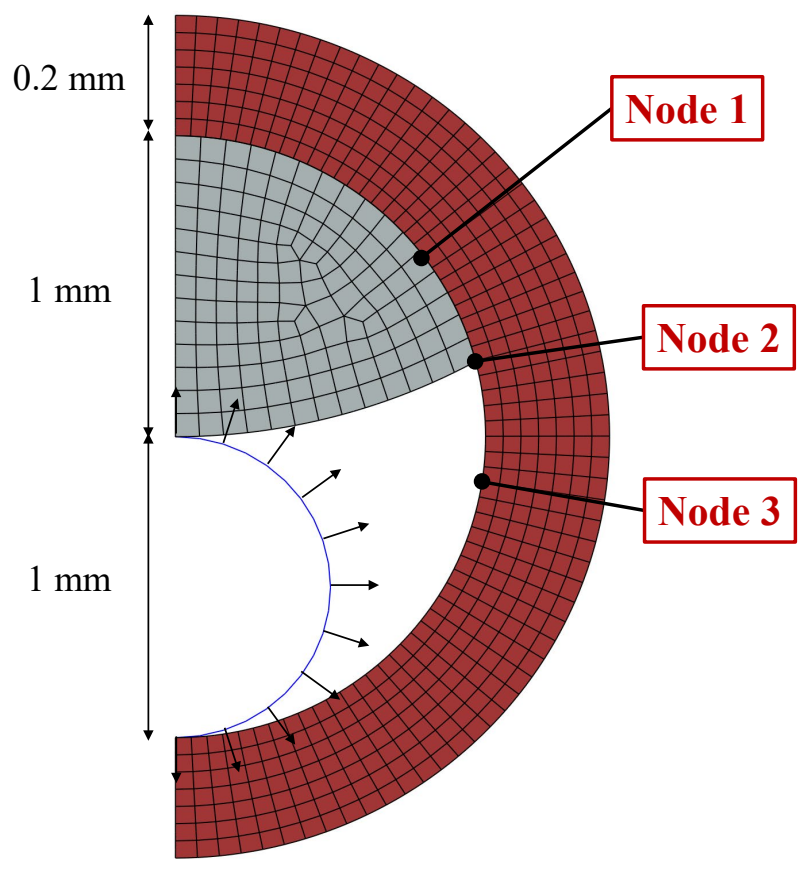

(b)

Figure 10: Reference model in the radial dilatation of an idealized artery. (a) Geometry, dimensions and boundary conditions; and (b) FEM mesh. 


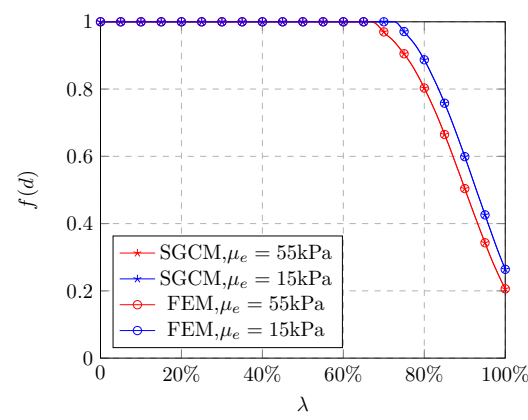

(a)

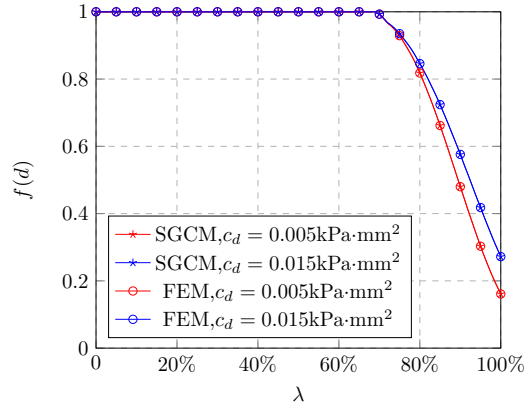

(b)

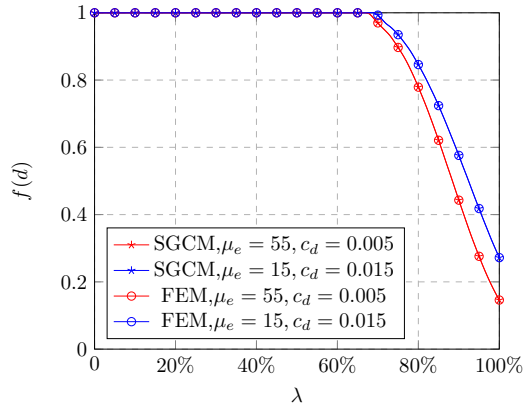

(c)

Figure 11: The comparison of the results obtained by surrogate model with the SGCM and FEM. (a) The damage function $f(d)$ at Node 1 for Case 1, (b) The damage function $f(d)$ at Node 1 for Case 2, and (c) The damage function $f(d)$ at Node 1 for Case 3 . 
(a)
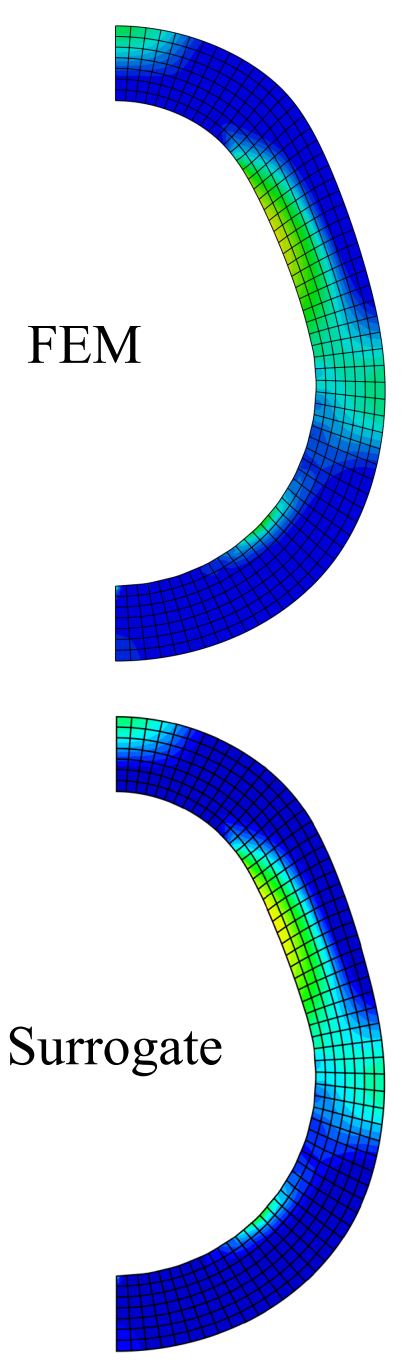

(b)
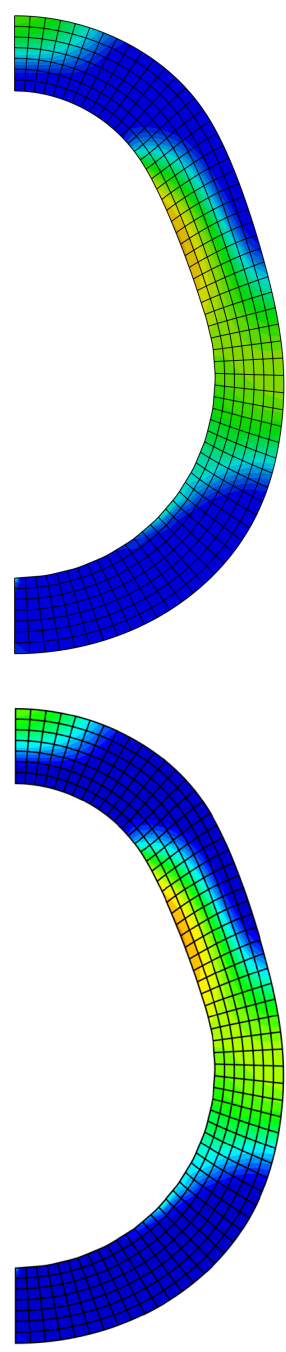

(c)
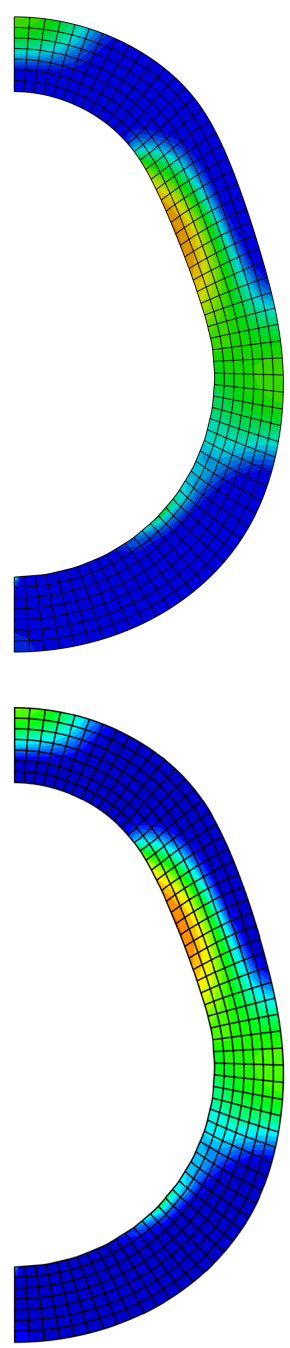

(d)

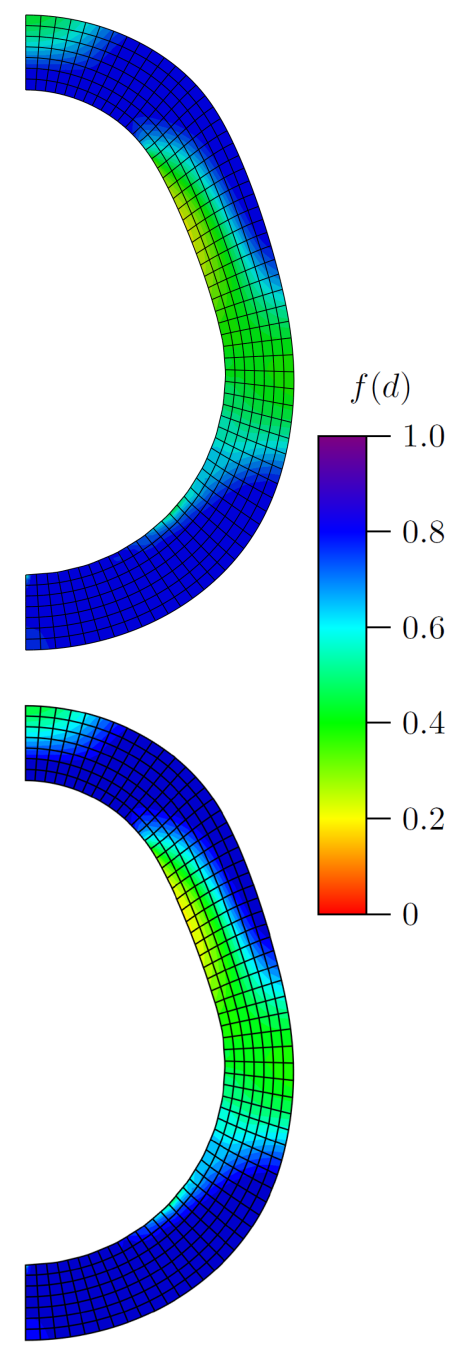

Figure 12: The contours of the damage function $f(d)$ obtained by the surrogate model with the SGCM and FEM for different shear modulus and the internal length scales when $\lambda=100 \%$. (a) $\mu_{e}=15 \mathrm{kPa}$, $c_{d}=0.01 \mathrm{kPa}^{-1} \cdot \mathrm{mm}^{2}$, (b) $\mu_{e}=55 \mathrm{kPa}, c_{d}=0.01 \mathrm{kPa}^{-1} \cdot \mathrm{mm}^{2}$, (c) $\mu_{e}=35 \mathrm{kPa}, c_{d}=0.005 \mathrm{kPa}^{-1} \cdot \mathrm{mm}^{2}$, and (d) $\mu_{e}=35 \mathrm{kPa}, c_{d}=0.015 \mathrm{kPa}^{-1} \cdot \mathrm{mm}^{2}$ 

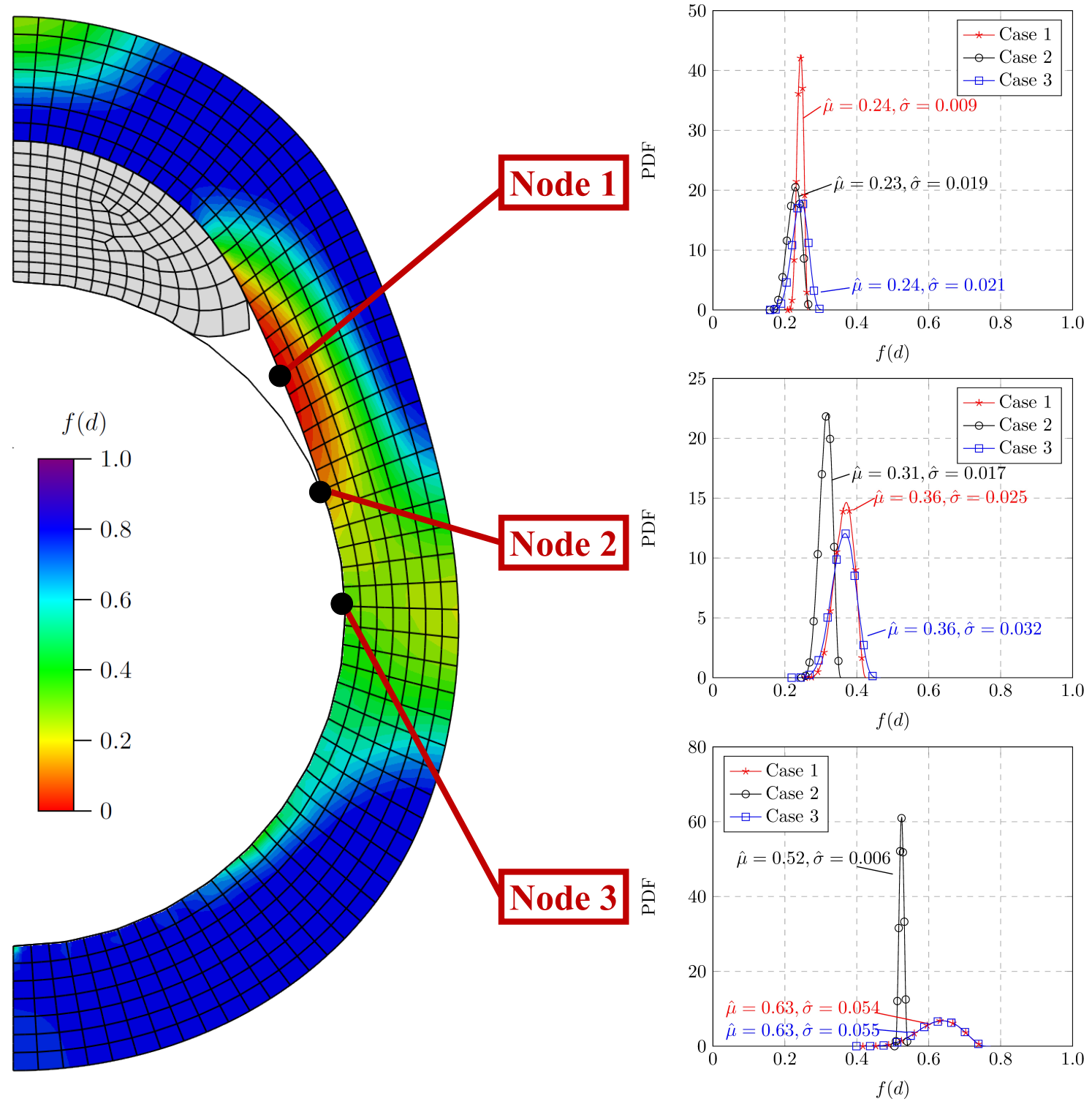

Figure 13: The PDF of the damage function $f(d)$ for different cases at different locations when $\lambda=100 \%$. 


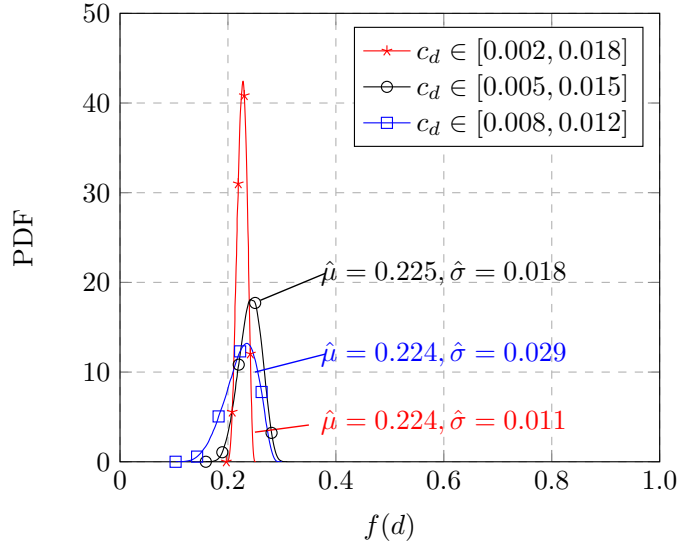

(a)

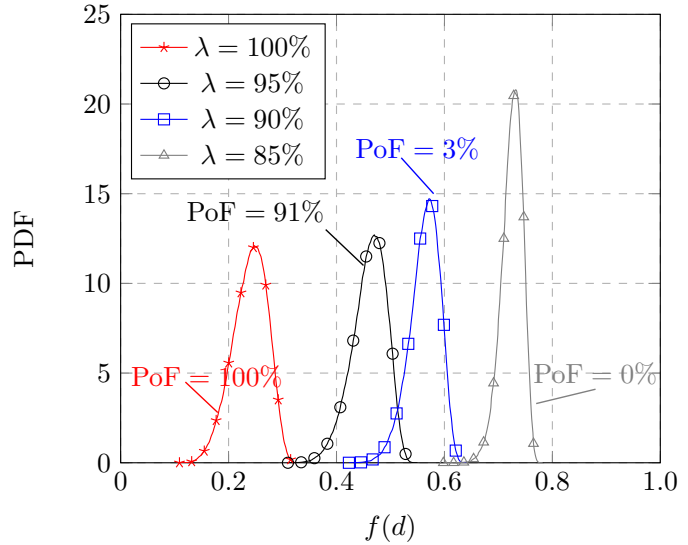

(b)

Figure 14: The PDFs of the damage function $f(d)$ for different situations at Node 1. (a) The PDFs of the damage function $f(d)$ for different internal length scales $c_{\mathrm{d}}$ when $\lambda=100 \%$, and (b) The PDFs of the damage function $f(d)$ for different $\lambda$. 